\title{
DYNAMICAL CONSTRAINTS ON THE ORIGIN OF HOT AND WARM JUPITERS WITH CLOSE FRIENDS
}

\author{
Fabio ANTONINI ${ }^{1}$, AdRian S. Hamers ${ }^{2}$, AND Yoram Lithwick ${ }^{1}$ \\ ${ }^{1}$ Center for Interdisciplinary Exploration and Research in Astrophysics (CIERA) and Department of Physics and Astrophysics, \\ Northwestern University, Evanston, IL 60208, USA \\ ${ }^{2}$ Leiden Observatory, Niels Bohrweg 2, Leiden, 2333CA, The Netherlands \\ Received 2016 April 6; revised 2016 July 30; accepted 2016 August 12; published 2016 November 22
}

\begin{abstract}
Gas giants orbiting their host star within the ice line are thought to have migrated to their current locations from farther out. Here we consider the origin and dynamical evolution of observed Jupiters, focusing on hot and warm Jupiters with outer friends. We show that the majority of the observed Jupiter pairs (20 out of 24) are dynamically unstable if the inner planet is placed at $\gtrsim 1$ au distance from the stellar host. This finding is at odds with formation theories that invoke the migration of such planets from semimajor axes $\gtrsim 1$ au due to secular dynamical processes (e.g., secular chaos, Lidov-Kozai [LK] oscillations) coupled with tidal dissipation. In fact, the results of $N$-body integrations show that the evolution of dynamically unstable systems does not lead to tidal migration but rather to planet ejections and collisions with the host star. This and other arguments lead us to suggest that most of the observed planets with a companion could not have been transported from farther out through secular migration processes. More generally, by using a combination of numerical and analytic techniques, we show that the high- $e$ LK migration scenario can only account for less than $10 \%$ of all gas giants observed between 0.1 and 1 au. Simulations of multiplanet systems support this result. Our study indicates that rather than starting on highly eccentric orbits with orbital periods above $1 \mathrm{yr}$, these "warm" Jupiters are more likely to have reached the region where they are observed today without having experienced significant tidal dissipation.
\end{abstract}

Key words: planetary systems - planets and satellites: dynamical evolution and stability - planets and satellites: gaseous planets

\section{INTRODUCTION}

The observed abundance of Jupiter-size planets orbiting interior to the ice line around their stars poses a challenge to our current understanding of planet formation (Wright et al. 2012). Close-in planets $(\lesssim 1 \mathrm{au})$ are typically thought to have formed beyond the ice line, where large, icy cores can grow and accrete, and to have moved within 1 au later on. Possible mechanisms for migration invoke gentle disk migration (e.g., Goldreich \& Tremaine 1980; Lin et al. 1996) or tidal interaction with the stellar host that gradually removes energy from the planet orbit. In the latter model the interaction with an external perturber (e.g., a star, a planet companion) moves the planet onto a highly eccentric orbit so that efficient tidal circularization can take place (e.g., Rasio \& Ford 1996; Wu \& Murray 2003; Naoz et al. 2011; Wu \& Lithwick 2011).

Migration scenarios must account for the existence of both hot Jupiters (HJs; gas giants orbiting within 0.1 au of their host stars) and warm Jupiters (WJs; orbiting in the region from 0.1 to $1 \mathrm{au}$ ). WJs are giant planets observed within the so-called period "valley," corresponding to the dip in the giant planet orbital period distribution from roughly $P=10$ to 100 days (Santerne et al. 2016). Thus, WJs are interior to both the ice line and the observed pileup of giant planets beyond $1 \mathrm{au}$. While most HJs have nearly zero eccentricities, WJs have a range of eccentricities, with a few being on highly eccentric ( $\gtrsim 0.8$ ) orbits (e.g., Dawson \& Murray-Clay 2013).

Although HJs and WJs appear to be separated in their period and eccentricity distributions, it has been suggested that they might share a common origin. A possibility is that both HJs and WJs migrated inward through high-e migration processes such as secular chaos and Lidov-Kozai (LK) cycles coupled with tidal friction (e.g., Dawson \& Chiang 2014; Dong et al. 2014; Frewen \& Hansen 2016). In this scenario the HJ orbits have been fully circularized by tidal friction, while WJs are still on their way to becoming HJs and are experiencing largeamplitude eccentricity oscillations induced by an external perturber. In fact, most gas giants observed in the period valley have observed eccentricities that are too small for significant tidal evolution, but this can be understood if they are currently near the low-e phase of an LK cycle, while periodically attaining high eccentricities and thereby experiencing significant tidal dissipation.

In this paper we examine whether high-e migration models are consistent with the observed properties of the WJ population. We based our analysis on (mostly) radial velocity data from the exoplanet database at http://exoplanets.org (Wright et al. 2011). In particular, we focus on planets that have a detected outer companion and that orbit their stellar host interior to $1 \mathrm{au}$. We use both a high-precision three-body integrator and an orbit-average secular code to produce synthetic populations of migrating planets. By comparing our results to observations, we are able to address whether the giant planets observed in the period valley could have formed through secular migration processes.

We find that secular processes do cause giant planets to migrate within the radial range 0.1-1 au; however, the orbital properties of the migrating planets are not consistent with what is observed. Our results are consistent with less than $10 \%$ of all gas giants observed in the period valley having migrated through tidal dissipation. We note that our results are somewhat complementary to those of Huang et al. (2016). These authors recently used Kepler transit data to show that HJs and WJs are distinct in their respective fractions of sub-Jovian companions. They found that HJs as a whole do not have any detectable inner or outer planetary companions with periods inward of 50 days. In stark contrast, half of the WJs in their sample have 
small companions. Motivated by this discovery and by additional arguments, Huang et al. (2016) proposed that a large fraction of WJs are formed in situ.

The paper is organized as follows. In Section 2 we consider all planets within 1 au that have a detected outer companion and address the dynamical stability of these systems. In Section 3 we describe our numerical methods. In Section 4 we describe the results of the $\mathrm{N}$-body integrations that we used to study the dynamical evolution of systems close to the stability boundary. In Sections 5 and 6 we study the dynamical evolution of planets undergoing secular migration and their resulting orbital distribution. Section 7 summarizes our main results.

\section{STABILITY}

In this section we discuss the stability of observed Jupiter pairs hosting HJs and WJs. In particular, we examine whether the $\mathrm{HJ}$ or $\mathrm{WJ}$ could have reached its current orbit via high-e migration, or whether its having a highe and $a \sim 1$ au in the past would instead have made the system dynamically unstable.

The majority of WJs are far enough from their stellar hosts that they are not expected to experience significant tidal dissipation. However, if the eccentricities of the WJs are experiencing large-amplitude LK oscillations induced by an external perturber, then they might be currently at the low-e phase of an LK cycle. Over a secular timescale they might access a periapsis separation such that $a_{1}\left(1-e_{1}^{2}\right)<a_{\mathrm{cr}} \approx 0.1 \mathrm{au}$, within which tidal dissipation will cause efficient migration. In this scenario the WJs have to be accompanied by a strong enough perturber to overcome Schwarzschild precession. Note that at $\sim 0.1$ au, the additional precession due to tides is negligible compared to Schwarzschild precession for typical hosts.

Dong et al. (2014) consider the secular migration scenario for WJs. At the quadrupole level of approximation they derive an analytic upper limit on the outer perturber separation by requiring the $\mathrm{WJ}$ to reach $a_{1}\left(1-e_{1}^{2}\right)<a_{\mathrm{cr}}$ during LK oscillations:

$$
\begin{aligned}
\frac{a_{2} \sqrt{1-e_{2}^{2}}}{a_{1}} \lesssim & \left(\frac{8 G M_{\star}}{c^{2} a_{1}}\right)^{-1 / 3}\left(\frac{M_{\star}}{M_{2}}\right)^{-1 / 3} \\
& \times\left[2 e_{1}^{2}+3\left(1-\frac{a_{\mathrm{cr}}}{a_{1}}\right)\right]^{1 / 3} \\
& \times\left(\sqrt{\frac{a_{1}}{a_{\mathrm{cr}}}}-\frac{1}{\sqrt{1-e_{1}^{2}}}\right)^{-1 / 3},
\end{aligned}
$$

where $M_{\star}$ is the mass of the host star, $M_{2}$ is the mass of the outer perturber, and $a_{1}\left(a_{2}\right)$ and $e_{1}\left(a_{2}\right)$ are the semimajor axis and eccentricity of the inner (outer) planet, respectively. In the limit $a_{1} \gg a_{\mathrm{cr}}$ and $e_{1} \rightarrow 0$, Equation (1) becomes

$$
\begin{aligned}
\frac{a_{2} \sqrt{1-e_{2}^{2}}}{a_{1}} & \lesssim\left(\frac{8 G M_{\star}}{c^{2} \sqrt{a_{1} a_{\mathrm{cr}}}}\right)^{-1 / 3}\left(\frac{M_{\star}}{M_{2}}\right)^{-1 / 3} \\
\approx & 20\left(\frac{M_{2}}{M_{\text {Jupiter }}}\right)^{1 / 3} \times\left(\frac{M_{\star}}{M_{\odot}}\right)^{-2 / 3} \\
& \times\left(\frac{a_{1}}{0.2 \mathrm{au}}\right)^{1 / 6}\left(\frac{a_{\mathrm{cr}}}{0.1 \mathrm{au}}\right)^{1 / 6}
\end{aligned}
$$

Using the above equations, Dong et al. (2014) concluded that "for a WJ at 0.2 au, a Jupiter perturber is required at $\lesssim 3$ au."

Previous work did not consider the stability of the initial configurations that can lead to the formation of a WJ in the secular migration scenario. In addition to the condition given by Equation (1), one must require the planetary system to be dynamically stable in its initial configuration, i.e., before tidal dissipation has significantly shrunk the orbit of the inner planet.

We compare the observed system configurations to various stability criteria. We consider the criterion (Petrovich 2015b)

$$
\frac{a_{2}\left(1-e_{2}\right)}{a_{1}\left(1+e_{1}\right)}>2.4\left[\max \left(\frac{M_{2}}{M_{\star}}, \frac{M_{1}}{M_{\star}}\right)\right]^{1 / 3}\left(\frac{a_{2}}{a_{1}}\right)^{1 / 2}+1.15,
$$

which is applicable to planet-star mass ratios $10^{-4}-10^{-2}$ and mutual inclinations up to $40^{\circ}$. This criterion is essentially equivalent to that of Eggleton \& Kiseleva (1995):

$$
\begin{aligned}
\frac{a_{2}\left(1-e_{2}\right)}{a_{1}\left(1+e_{1}\right)}> & 1+3.7\left(\frac{M_{2}}{M_{\star}}\right)^{1 / 3}+\frac{2.2}{1+\left(M_{2} / M_{\star}\right)^{-1 / 3}} \\
& +1.4\left(\frac{M_{1}}{M_{\star}}\right)^{1 / 3} \frac{\left(M_{2} / M_{\star}\right)^{-1 / 3}-1}{1+\left(M_{2} / M_{\star}\right)^{-1 / 3}} .
\end{aligned}
$$

Systems that do not satisfy the conditions given by Equations (3) and (4) are expected to be unstable, leading to either ejections or collisions. It can be shown by combining Equations (2) and (3) and taking the limit $e_{1} \rightarrow 1$ in the latter equation that for a Jupiter-mass perturber there are no stable configurations that allow the formation of a WJ at $\lesssim 0.3$ au.

Another often adopted stability criterion is that of Mardling \& Aarseth (2001),

$$
\frac{a_{2}\left(1-e_{2}\right)}{a_{1}}>2.8\left[\left(1+\frac{M_{2}}{M_{\star}}\right) \frac{1+e_{2}}{\left(1-e_{2}\right)^{1 / 2}}\right]^{2 / 5} .
$$

Note that the Mardling \& Aarseth (2001) criterion does not include a dependence on the inner-planet orbital eccentricity and was derived for cases in which the mass ratio between the inner and outer binary is not much different from unity. For these reasons, we consider Equations (3) and (4) more accurate for the two-planet systems we are considering. The results of our simulations confirm this.

In Figure 1 we compute the stability boundaries defined above by adopting the observed orbital parameters of HJs and WJs with a detected companion. The full sample of planets we considered is presented in Table 1. We selected systems with two giant planets and that host a Jupiter planet with mass $M_{1} \sin i \geqslant 0.5 M_{\text {Jupiter }}$ and semimajor axis $<1$ au. The left panel shows the inner Jupiter semimajor axis as a function of the critical inner-planet semimajor axis that would render the system unstable according to Equations (3) and (5). At larger semimajor axis the system will be unstable, and any secular process leading to high- $e$ migration is likely to be suppressed. Since a secular migration scenario requires the inner Jupiter to have initially an extremely large eccentricity, we take the limit $e_{1} \rightarrow 1$ when evaluating Equation (3). Also, we added a factor of 0.5 to right-hand side of Equation (3), which, according to Petrovich (2015b), corresponds approximately to a 95\% chance for a system to be unstable over $10^{8} \mathrm{yr}$ of evolution. 

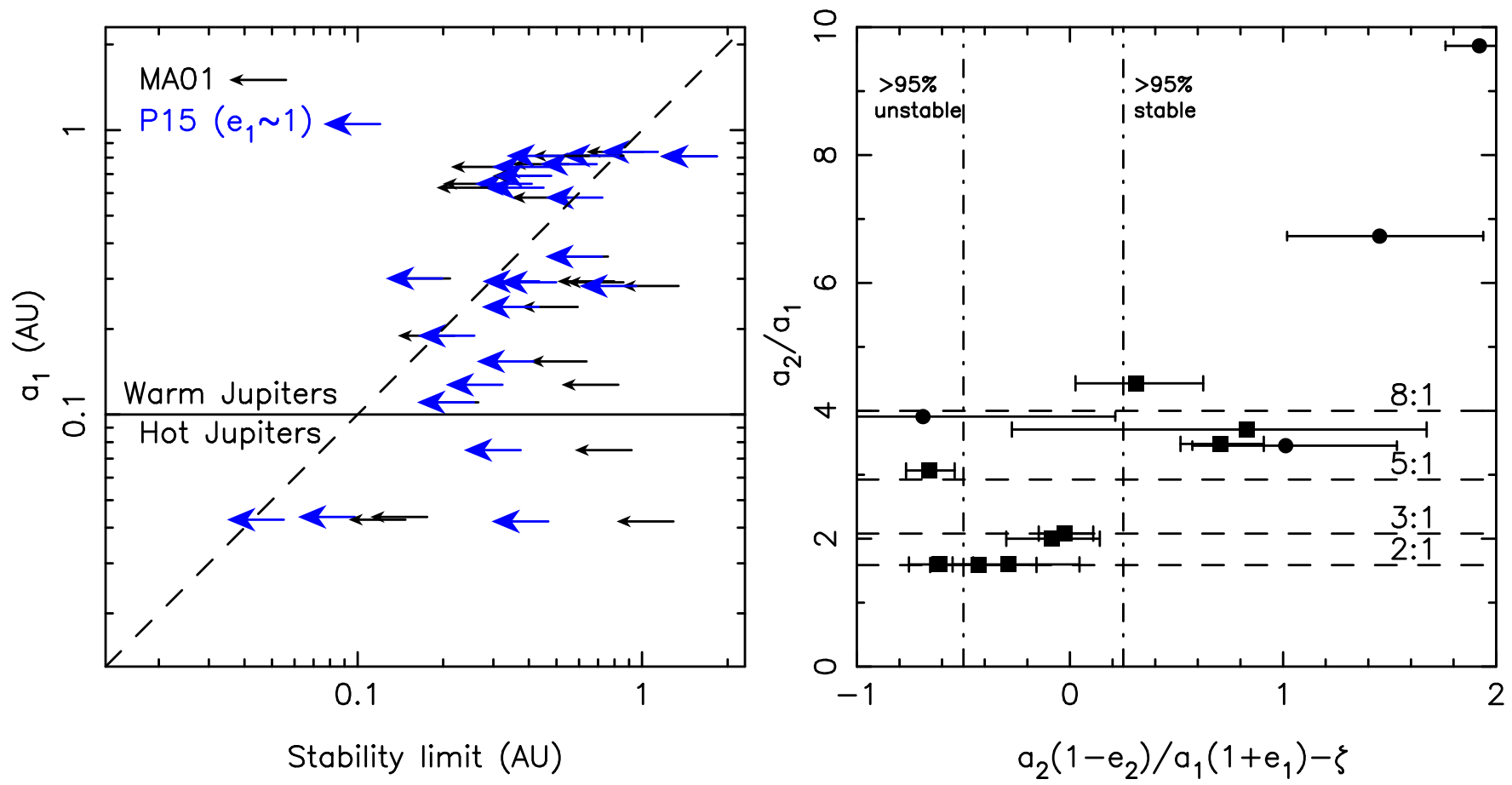

Figure 1. Left panel: semimajor axis of HJs and WJs, with observed companions plotted as a function of the inner-planet semimajor axis above which the system will be unstable. The inner planet must have been to the left of the tip of each arrow during its high- $e$ migration (assuming that it formed via high- $e$ migration); otherwise, it would have been dynamically unstable according to Equation (5) (black arrows) and Equation (3) (blue arrows). The stability limit imposed by Equation (3) was computed taking the limit $e_{1} \rightarrow 1$ and adding a factor of 0.5 to the right-hand side, which approximately corresponds to a $95 \%$ chance for a system to be unstable over $10^{8} \mathrm{yr}$ of evolution. Systems that are at the left of the dashed line are dynamically unstable according to the stability criteria we considered. Right panel: stability boundary in Equation (3) as a function of the semimajor-axis ratio $a_{2} / a_{1}$ for our sample of two-planet systems; here $\zeta=2.4\left[\max \left(\mu_{2}, \mu_{1}\right)\right]^{1 / 3} \sqrt{a_{2} / a_{1}}+1.15$. The vertical dot-dashed line indicates the region for which $>95 \%$ of the systems to the left (right) are expected to be unstable (stable). The horizontal dashed lines indicate the position of some of the strongest mean motion resonances. Squares (circles) are systems with semimajor axis $a_{1} \geqslant 0.5 \mathrm{au}(\leqslant 0.5 \mathrm{au})$.

From the left panel of Figure 1 we see that most Jupiters at $0.6 \leqslant a_{1} \leqslant 0.8$ au are close to or above the stability boundary defined by the dashed line. Clearly this simple fact is difficult to reconcile with a high-e migration model for these planets, suggesting that such systems are unlikely to have experienced significant tidal migration from farther out. Note that in Figure 1 the stability boundaries were computed using the minimum mass for the planets. If the planet orbits were significantly tilted with respect to the line of sight, the planet masses could be significantly larger, which will further push the stability boundary toward smaller semimajor axes.

In the region 0.1 au $\leqslant a_{1} \leqslant 0.6$ au the two adopted stability criteria start to give somewhat different values for the limiting initial $a_{1}$ implied by our stability argument. According to Equation (3), 9 out of $10 \mathrm{WJ}$ s within 0.6 au could not have migrated from $a_{1} \gtrsim 1 \mathrm{au}$, as they would have been dynamically unstable otherwise. According to Equation (5) instead, 8 out of 10 WJs within 0.6 au would be dynamically unstable at $a_{1} \gtrsim 1 \mathrm{au}$, suggesting that they could not have migrated from these distances. Two (HAT-P-13 and Kepler-424) of the four HJs with known companions have very strong constraints on their maximum separation required by stability, implying that if they formed through secular migration, they must have been initially at $\lesssim 0.2$ au.

A possibility is that WJs formed by interactions with a planetary companion and began tidal circularization interior to $1 \mathrm{au}$ after multiple scatterings. However, among the $10 \mathrm{WJ}$ detected within $\lesssim 0.5 \mathrm{au}$, only 2 are above the stability boundary given by Equation (3) when setting $a_{1}=0.6$ au and taking the limit $e_{1} \rightarrow 1$; these planets are HD 37605c and
HD 163607b. There are also a few systems that at $a_{1}=0.6$ au would be classified as unstable according to Equation (3) but are just above the stability boundary defined by Equation (5). These systems are HD 38529, HD 74156, HD13908, and HD 168443. Further dynamical constraints on a possible high-e migration scenario for some of these systems are presented in the next sections.

In the right panel of Figure 1 we show the stability boundary in Equation (3) for our sample of two-planet systems. We also identify the region where $95 \%$ of the systems according to Equation (3) would be unstable after $10^{8} \mathrm{yr}$ of evolution. Surprisingly, some of these planets appear to be well inside the dynamically unstable region. As also noted by Petrovich (2015b), however, the stability of these systems might be promoted by mean motion resonances (some of which are indicated in Figure 1). In any case, it is hard to imagine how these planets could have been transported from farther out through secular migration processes.

The stability analysis shown in Figure 1 suggests that most WJs and HJs with observed companions cannot have migrated to their current location via tidal dissipation from $a_{1} \approx 1$ au. This idea is further explored and supported by the analysis presented in the following sections.

In Table 1 we give the orbital parameters of observed twoplanet systems with an inner WJ or HJ and summarize the results of our stability analysis. Importantly, and contrary to previous work (Dawson \& Chiang 2014), we note that our analysis disfavors a high-migration origin for most WJs with a companion, including those having a finite orbital eccentricity $\left(e_{1} \gtrsim 0.2\right)$. 
Table 1

Observed Orbital Elements of Detected Systems Comprising Warm $(0.1 \leqslant a \leqslant 1 \mathrm{au})$ and Hot $(a<0.1 \mathrm{au})$ Jupiters and Their Close Friend

\begin{tabular}{|c|c|c|c|c|c|c|c|c|}
\hline System & $\begin{array}{c}a_{1} \\
(\mathrm{au})\end{array}$ & $e_{1}$ & $\begin{array}{l}M_{1} \sin i_{1} \\
\left(M_{\text {Jupiter }}\right)\end{array}$ & $\begin{array}{c}a_{2} \\
(\mathrm{au})\end{array}$ & $e_{2}$ & $\begin{array}{l}M_{2} \sin i_{2} \\
\left(M_{\text {Jupiter }}\right)\end{array}$ & $\begin{array}{l}r_{\mathrm{cr}} \\
(\mathrm{au})\end{array}$ & $\begin{array}{l}\text { High-e } \\
\text { Migration }\end{array}$ \\
\hline HD 82943 & $0.742 \pm 0.0129$ & $0.425 \pm 0.03$ & $1.59 \pm 0.103$ & $1.185 \pm 0.022$ & $0.203 \pm 0.065$ & $1.589 \pm 0.097$ & 0.50 & $x \times$ \\
\hline HD 12661 & $0.838 \pm 0.0177$ & $0.3768 \pm 0.0077$ & $2.34 \pm 0.101$ & $2.919 \pm 0.064$ & $0.031 \pm 0.022$ & $1.949 \pm 0.092$ & 1.19 & $\checkmark$ \\
\hline HD 169830 & $0.813 \pm 0.0136$ & $0.310 \pm 0.01$ & $2.89 \pm 0.102$ & $3.60 \pm 0.35$ & $0.330 \pm 0.02$ & $4.06 \pm 0.35$ & 0.87 & $x$ \\
\hline HD 207832 & $0.570 \pm 0.02$ & $0.13 \pm 0.05$ & $0.564 \pm 0.065$ & $2.11 \pm 0.1$ & $0.27 \pm 0.1$ & $0.73 \pm 0.161$ & 0.73 & $\times$ \\
\hline HD 73526 & $0.647 \pm 0.011$ & $0.190 \pm 0.05$ & $2.86 \pm 0.172$ & $1.028 \pm 0.0177$ & $0.140 \pm 0.09$ & $2.42 \pm 0.167$ & 0.43 & $x \times$ \\
\hline HD 155358 & $0.627 \pm 0.0168$ & $0.170 \pm 0.03$ & $0.819 \pm 0.068$ & $1.001 \pm 0.027$ & $0.16 \pm 0.1$ & $0.807 \pm 0.056$ & 0.47 & $\times x$ \\
\hline HD 202206 & $0.812 \pm 0.0164$ & $0.4350 \pm 0.001$ & $16.82 \pm 0.68$ & $2.490 \pm 0.055$ & $0.267 \pm 0.021$ & $2.33 \pm 0.127$ & 0.65 & $x \times$ \\
\hline HD 60532 & $0.759 \pm 0.0176$ & $0.280 \pm 0.03$ & $1.035 \pm 0.069$ & $1.580 \pm 0.04$ & $0.020 \pm 0.02$ & $2.46 \pm 0.146$ & 0.73 & $x \times$ \\
\hline HD 134987 & $0.808 \pm 0.016$ & $0.2330 \pm 0.002$ & $1.563 \pm 0.062$ & $5.83 \pm 0.33$ & $0.120 \pm 0.02$ & $0.805 \pm 0.046$ & 1.93 & $\checkmark$ \\
\hline HD 37605 & $0.283 \pm 0.047$ & $0.6767 \pm 0.0019$ & $2.80 \pm 0.93$ & $3.82 \pm 0.64$ & $0.013 \pm 0.013$ & $3.4 \pm 1.12$ & 1.34 & $\checkmark$ \\
\hline HD 163607 & $0.3592 \pm 0.006$ & $0.730 \pm 0.02$ & $0.769 \pm 0.041$ & $2.418 \pm 0.041$ & $0.120 \pm 006$ & $2.29 \pm 0.108$ & 0.76 & $\times$ \\
\hline HD 147018 & $0.2389 \pm 0.004$ & $0.486 \pm 0.0081$ & $2.127 \pm 0.076$ & $1.923 \pm 0.039$ & $0.133 \pm 0.011$ & $6.59 \pm 0.29$ & 0.62 & $\times$ \\
\hline HD 74156 & $0.2915 \pm 0.0049$ & $0.630 \pm 0.01$ & $1.773 \pm 0.09$ & $3.900 \pm 0.067$ & $0.380 \pm 0.02$ & $8.25 \pm 0.36$ & 0.86 & $\times$ \\
\hline HD 13908 & $0.1538 \pm 0.0026$ & $0.046 \pm 0.022$ & $0.865 \pm 0.035$ & $2.034 \pm 0.042$ & $0.120 \pm 0.02$ & $5.13 \pm 0.25$ & 0.64 & $\times /$ \\
\hline HD 168443 & $0.2939 \pm 0.0049$ & $0.529 \pm 0.024$ & $7.70 \pm 0.29$ & $2.853 \pm 0.048$ & $0.2113 \pm 0.0017$ & $17.39 \pm 0.58$ & 0.80 & $\times$ \\
\hline HD 159243 & $0.1104 \pm 0.0018$ & $0.020 \pm 0.018$ & $1.130 \pm 0.05$ & $0.805 \pm 0.0171$ & $0.075 \pm 0.05$ & $1.90 \pm 0.13$ & 0.27 & $\times /$ \\
\hline HD 38529 & $0.1272 \pm 0.0021$ & $0.244 \pm 0.028$ & $0.803 \pm 0.033$ & $3.600 \pm 0.06$ & $0.3551 \pm 0.0074$ & $12.26 \pm 0.42$ & 0.83 & $\times 1$ \\
\hline HD 9446 & $0.1892 \pm 0.0063$ & $0.200 \pm 0.027$ & $0.699 \pm 0.065$ & $0.654 \pm 0.022$ & $0.060 \pm 0.06$ & $1.82 \pm 0.172$ & 0.27 & $\times /$ \\
\hline \multicolumn{9}{|l|}{ TYC-1422 } \\
\hline$-614-1$ & $0.689 \pm 0.036$ & $0.06 \pm 0.02$ & $2.5 \pm 0.4$ & $1.396 \pm 0.073$ & $0.048 \pm 0.014$ & $10 \pm 1$ & 0.51 & $x \times$ \\
\hline $\mathrm{K}-432$ & $0.301 \pm 0.065$ & $0.5134 \pm 0.0089$ & $5.5 \pm 2.4$ & $1.18 \pm 0.25$ & $0.498 \pm 0.059$ & $2.4 \pm 1.04$ & 0.21 & $x \times$ \\
\hline $\mathrm{K}-424$ & $0.04365 \pm 0.00078$ & $0.002 \pm 0.071$ & $1.034 \pm 0.099$ & $0.724 \pm 0.0137$ & $0.319 \pm 0.081$ & $7.04 \pm 0.58$ & 0.18 & $\times$ \\
\hline HAT-P-13 & $0.04269 \pm 0.00087$ & $0.0133 \pm 0.0041$ & $0.851 \pm 0.035$ & $1.226 \pm 0.025$ & $0.6616 \pm 0.0054$ & $14.27 \pm 0.69$ & 0.14 & $\times$ \\
\hline HD 217107 & $0.0750 \pm 0.00125$ & $0.1267 \pm 0.0052$ & $1.401 \pm 0.048$ & $5.33 \pm 0.2$ & $0.517 \pm 0.033$ & $2.62 \pm 0.15$ & 0.91 & $\checkmark$ \\
\hline HD 187123 & $0.04209 \pm 0.0007$ & $0.0103 \pm 0.0059$ & $0.510 \pm 0.0173$ & $4.83 \pm 0.37$ & $0.252 \pm 0.033$ & $1.94 \pm 0.152$ & 1.30 & $\checkmark$ \\
\hline
\end{tabular}

Note. Only systems with two giant planets were considered. Orbital parameters of known WJs and HJs with a detected Jupiter companion are given. We selected systems with two giant planets, hosting a Jupiter planet with mass $M_{1} \sin i \geqslant 0.5 M_{\text {Jupiter }}$ and semimajor axis $<1$ au. The value of $r_{\text {cr }}$ is the maximum value of the innerplanet semimajor axis above which the two-planet system will be dynamically unstable, which we computed as the maximum value between the two stability boundaries obtained via Equations (3) and (5). The stability limit imposed by Equation (3) was computed taking the limit $e_{1} \rightarrow 1$ and adding a factor of 0.5 to the right-hand side, which corresponds to a 95\% chance for a system to be unstable over $10^{8}$ yr. Systems with $r_{\text {cr }} \lesssim 1$ au $\left(r_{\text {cr }} \lesssim a_{1}\right)$ are indicated with a " $\times$ " (“ $\times \times$ ") symbol in the last column. Our stability analysis disfavors a high-migration scenario for the formation of these systems. Systems with a low eccentricity, $e_{1} \leqslant 0.3$, at $0.1 \leqslant a_{1} \leqslant 0.3$ au are indicated with a "/" symbol. Our analysis of Section 5.1 disfavors a high-migration scenario for these systems as well. Systems for which our study does not rule out a secular migration origin are indicated with a " $\checkmark$ " symbol. The observations reported at http://exoplanets.org (Wright et al. 2011) include data from Mayor et al. (2004), Correia et al. (2005), Tinney et al. (2006), Desort et al. (2008), Wright et al. (2009), Ségransan et al. (2010), Hébrard et al. (2010), Winn et al. (2010), Jones et al. (2010), Pilyavsky et al. (2011), Meschiari et al. (2011), Wang et al. (2012), Haghighipour et al. (2012), Giguere et al. (2012), Robertson et al. (2012), Tan et al. (2013), Endl et al. (2014), Moutou et al. (2014), Niedzielski et al. (2015), and Quinn et al. (2015).

\section{NUMERICAL METHODS AND TEST CASES}

In what follows we study the evolution of Jupiter-like planets around a solar-like star induced by the gravitational interaction with an outer Jupiter companion. Our goal is to put constraints on the origin of some of the observed Jupiters within $1 \mathrm{au}$, focusing mostly on planets within the period valley that have a close companion. In order to do so, we use two numerical approaches: (i) direct $\mathrm{N}$-body integrations of the equations of motion and (ii) integrations of the orbit-averaged secular equations of motion. In both cases we included terms in the equations of motion that account for Schwarzschild precession, apsidal precession due to tidal bulges, and tidal dissipation.

The direct integrations presented below were performed using ARCHAIN (Mikkola \& Merritt 2008). ARCHAIN employs an algorithmically regularized chain structure and the time-transformed leapfrog scheme, which allow us to integrate the evolution of the motion of arbitrarily tight binaries with arbitrary mass ratio with extremely high precision. The code includes post-Newtonian (PN) nondissipative 1PN and 2PN and dissipative 2.5PN corrections to all pair forces. To these we also added terms that account for precession induced by tidal bulges, as well as tidal dissipation. Velocity-dependent forces were implemented using the generalized midpoint method described in Mikkola \& Merritt (2006). The tidal perturbation force was set equal to (Hut 1981)

$$
\mathbf{F}=-G \frac{M_{\star} M_{1}}{r^{2}}\left[3 \frac{M_{\star}}{M_{1}}\left(\frac{R}{r}\right)^{5} k\left(1+3 \frac{\dot{r}}{r} \tau\right) \hat{r}\right],
$$

where $M_{1}$ is the mass of the planet and $R$ its radius, hereafter set equal to one Jupiter radius, $k$ (set to 0.28 ) is the apsidal motion constant, and $\tau$ is the constant time-lag factor. Hereafter we use $M_{\star}=1 M_{\odot}$.

The secular integrations performed in this paper make use of the standard octupole-level secular equations of motion of (Blaes et al. 2002, their Equations (11)-(17)), including terms accounting for relativistic precession. We added terms that describe apsidal precession induced by tidal bulges and tidal friction. The perturbing acceleration (6) causes a slow change of the orbital parameters. Following Socrates et al. (2012a), in the limit of high $e$, the orbit-average change rate corresponding 
to Equation (6) is

$$
\begin{gathered}
\frac{\dot{a}}{a}=-\frac{4059}{320 t_{D}} \sqrt{\frac{a_{F}}{a}}, \\
\frac{\dot{e}}{e}=\frac{\dot{a}}{a} \frac{\left(1-e^{2}\right)}{2 e^{2}},
\end{gathered}
$$

and

$$
\dot{\omega}=\frac{15\left[G\left(M_{\star}\right)\right]^{1 / 2}}{8 a_{1}^{13 / 2}} \frac{8+12 e_{1}^{2}+e_{1}^{4}}{\left(1-e_{1}^{2}\right)^{5}} \frac{M_{\star}}{M_{1}} k R^{5},
$$

where $a_{F}=a\left(1-e^{2}\right)$ and $t_{D}$ is the characteristic time for tidal dissipation,

$$
t_{D}=\frac{M_{1} a_{F}^{8}}{6 k \tau G M_{\star}^{2} R^{5}} .
$$

In our simulations we neglected any additional precession induced by the stellar host rotational bulge. For the cases considered here we find in fact that the precession due to tidal bulges is dominant and rotational bulges become important only for rapidly rotating stars with spin period less than $\approx 1$ day. Finally, we assume that the inner planet was disrupted by its host star tidal field if it crossed the Roche limit:

$$
a_{1}\left(1-e_{1}\right) \leqslant 0.01 \frac{R}{R_{\text {Jupiter }}}\left(\frac{M_{\star}}{M_{\odot}} \frac{M_{\text {Jupiter }}}{M_{1}}\right)^{1 / 3} \text { au. }
$$

The secular integrations are based on two levels of approximation being implemented: (i) double-orbit averaging and (ii) perturbation up to octupole order. More specifically, the orbit-average approximation, on which the Blaes et al. (2002) treatment is based, breaks down if (Antonini et al. 2014)

$$
\frac{a_{2}\left(1-e_{2}\right)}{a_{1}} \lesssim 0.2\left(\frac{M_{\odot}}{M_{\star}} \frac{M_{2}}{M_{\text {Jupiter }}}\right)^{1 / 3}\left(\frac{a_{1}}{a_{\mathrm{cr}}} 0.1\right)^{1 / 6} .
$$

For values of $a_{2} / a_{1}$ smaller than the one given by this last equation the orbital angular momentum of the inner planet can undergo oscillations on a timescale shorter than its orbital period. Clearly, this condition is never met for the two-planet systems considered here unless the orbits of the planets are crossing. Mean motion resonances are also neglected in our secular integrations. We note that for $a_{2} / a_{1} \approx 3$, their effect might not be fully negligible. The potential associated with $q$ thorder mean motion resonances has terms with amplitude $\sim e^{q}$, and since the eccentricities are order unity (especially for migrating planets), their effect might be significant. Moreover, even if the effect of mean motion resonances is negligible, the octupole-level expansion might not resolve the behavior of the $N$-body properly for low $a_{2} / a_{1}$ as more terms in the expansion might be needed. Although the simplifications implemented in our treatment should be taken with caution, the results of secular and direct integrations were compared for a number of initial conditions and found to give, in general, consistent results.

In Figure 2 we show two example cases. The top panel shows a three-body integration with initial conditions representing a possible progenitor for HD 37605c and initial mutual inclination $I=94^{\circ}$. The observed system consists of an eccentric $\left(e_{1}=0.68\right) \mathrm{WJ}$ at $a_{1}=0.28$ au and minimum mass
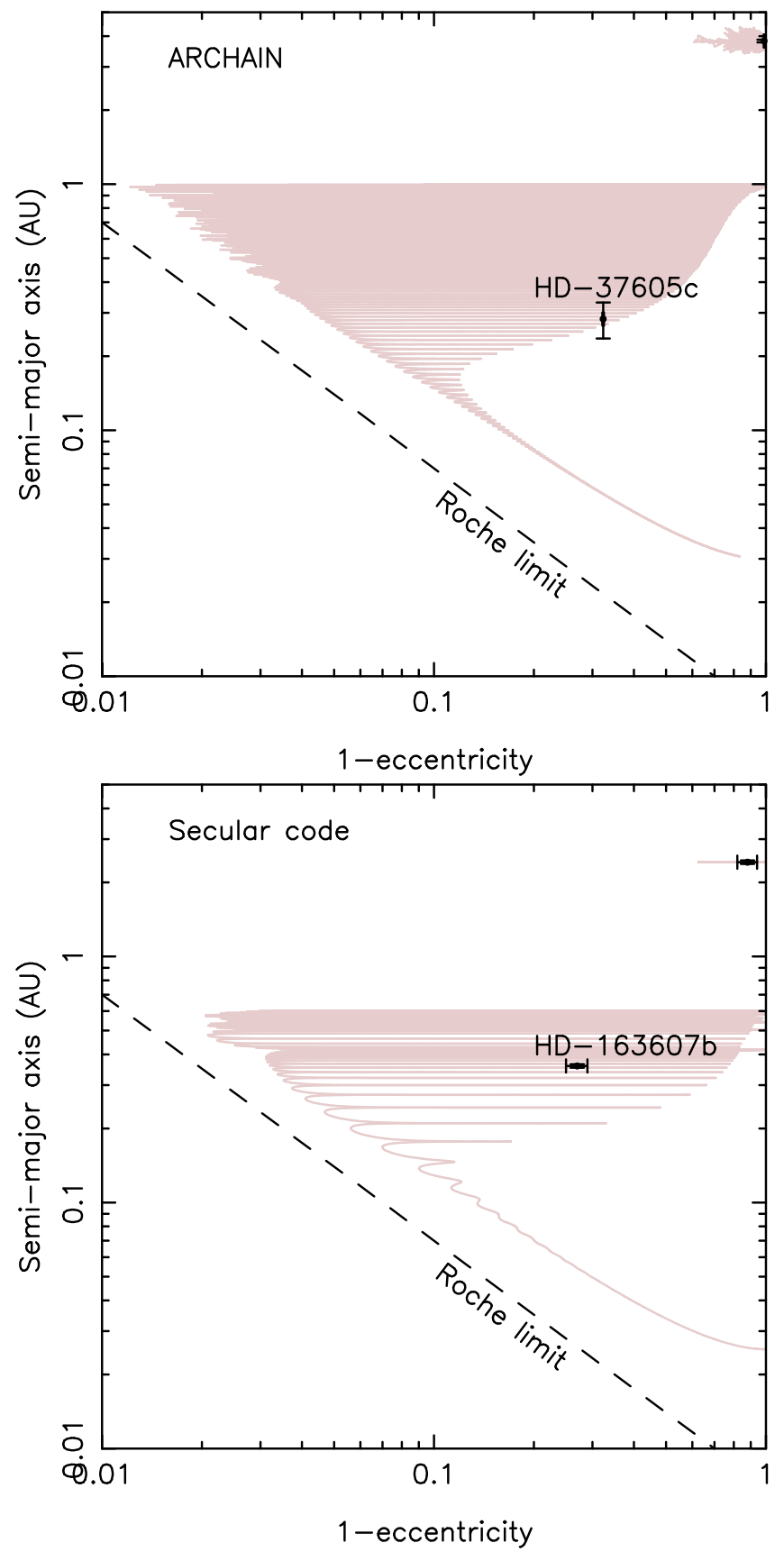

Figure 2. Evolution examples that lead to the formation of two-planet systems resembling the observed systems HD 37605 and HD 163607. In the bottom panel we evolved the system using the secular equations of motion, and in the top panel we used the direct integrator ARCHAIN. Dashed lines indicate the limit below which the planet will undergo Roche lobe overflow. In both cases the systems are initially stable according to Equation (3). The inner planet evolves to attain an orbit that is consistent with the orbits of the observed planets. These two systems represent therefore possible candidates for a secular migration origin, although our stability analysis suggests that HD 163607b could not have migrated from distances much larger than $\sim 0.6$ au.

$M_{1} \sin i=2.8 M_{\text {Jupiter }}$, with a companion at $a_{1}=3.8$ au and minimum mass $M_{2} \sin i=3.4 M_{\text {Jupiter }}$. According to our analysis in Section 2, this is the only two-planet system hosting a WJ within $\leqslant 0.5$ au that would be dynamically stable if we were to place the inner planet at 1 au on a highly eccentric orbit. Thus, HD 37605c is a possible candidate for a secular migration origin. The example in the top panel of Figure 2 

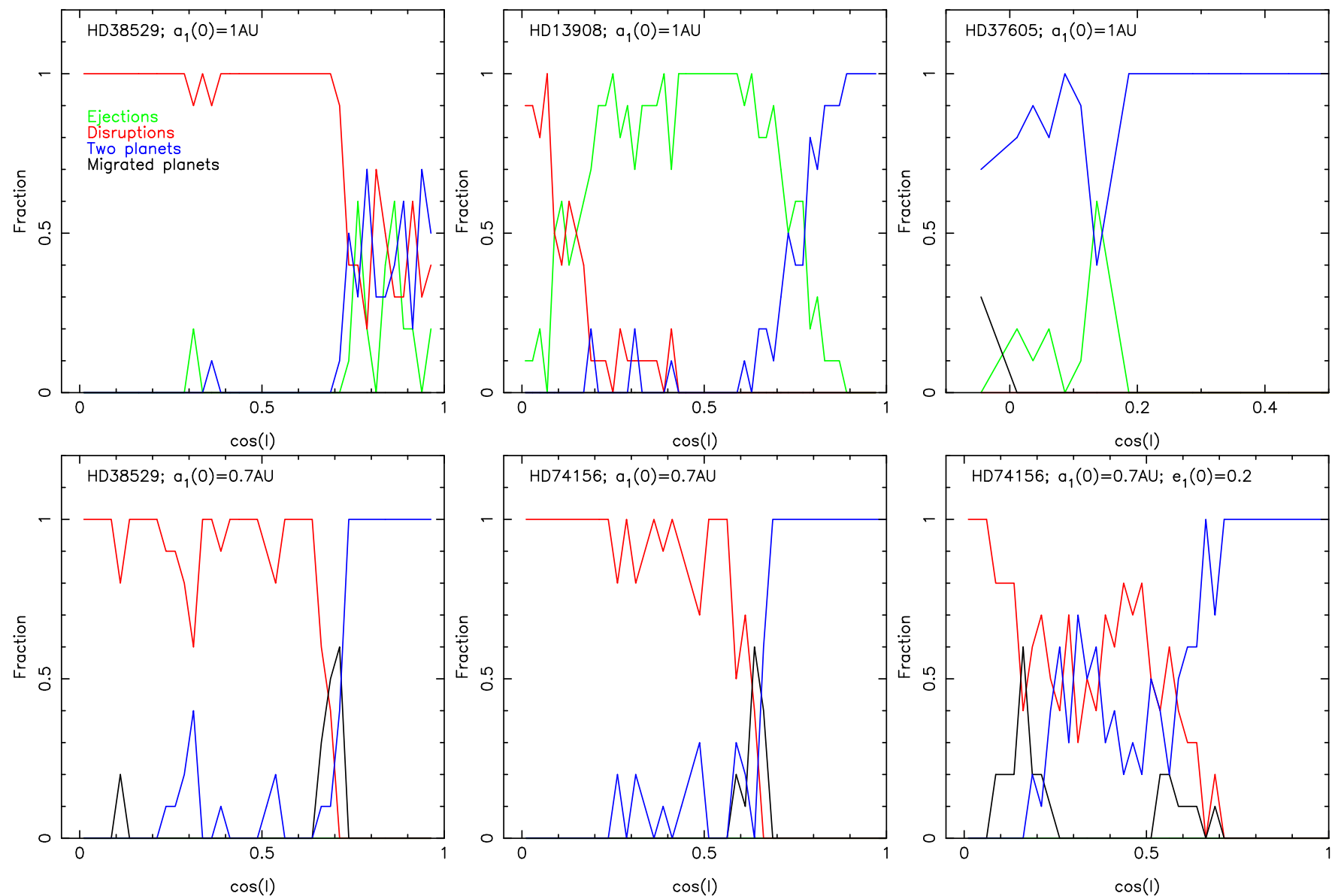

Figure 3. Results of the direct $N$-body integrations. In the top panels the inner planet was started at $a_{1}(0)=1$ au, while in the bottom panels it was started at $a_{1}(0)=0.7 \mathrm{au}$. The initial values of $a_{2}, e_{2}, M_{2}$, and $M_{1}$ are given in Table 1 for each system. Here we set the initial masses equal to the minimum mass given in the table. Note that no HJ or WJ is produced for configurations that violate the stability criteria of Section 2 . When the initial conditions are stable for large mutual inclinations (bottom panels), LK cycles combined with tidal friction lead to the formation of HJs and WJs. Systems in which one of the two planets is ejected during the simulation are indicated as "Ejections"; systems in which the inner planet had crossed its Roche limit are indicated as "Disruptions." "Migrated planets" are systems in which at the end of the simulation the innermost planet was at $a_{1} \leqslant 0.5 \mathrm{au}$. Systems that are stable and in which the innermost planet did not experience significant tidal dissipation $\left(a_{1}>0.5 \mathrm{au}\right)$ are indicated as "Two planets."

shows that this secular migration scenario is indeed a possibility for such a system. The inner planet starts at $a_{1}(0)=1 \mathrm{au}$ and evolves to become an HJ. During this transition, the inner-planet orbital semimajor axis and eccentricity take values that are consistent with the observed orbit of HD 37605c. Note that the amount of time the system spends in this region of parameter space depends on the efficiency of tidal dissipation, which in turn is regulated by the poorly constrained value of $\tau$ in Equation (6). However, the characteristic shape of the envelope within which the planet orbit evolves does not depend significantly on the assumed value of $\tau$ (see also Section 4).

For the bottom panel of Figure 2 we show a secular integration of a two-planet system with initial conditions that resemble the observed system HD 163607 and mutual inclination $I=84^{\circ}$. In this case we start the inner planet at $a_{1}(0)=0.6 \mathrm{au}$. The observed system consists of an eccentric $\left(e_{1}=0.73\right)$ WJ at $a_{1}=0.36$ au and minimum mass $M_{1} \sin i=0.77 M_{\text {Jupiter }}$, with a companion at $a_{1}=2.4$ au and minimum mass $M_{2} \sin i=2.3 M_{\text {Jupiter }}$. Even in this case the inner planet evolves through a region of parameter space that is consistent with the observed orbit of the planet HD $163607 \mathrm{~b}$.

\section{4. $N$-BODY SIMULATIONS: NEAR THE EDGE OF STABILITY}

In this section we consider the evolution of two-planet systems that are close to the stability boundary defined by Equation (3). In particular, we focus on systems with properties that resemble those of the two-planet systems HD 38529, HD 74156, and HD13908. (We specifically analyzed the stability of these three systems because their value of $r_{\text {cr }}$ in Table 1 is just below $1 \mathrm{au}$. Thus, given the uncertainty in the adopted stability criteria, it is unclear whether these systems will be actually unstable at $a_{1} \approx 1 \mathrm{au}$.) The results of these simulations are used to validate the stability criteria adopted above and our argument that systems that are dynamically unstable according to these criteria do not lead to tidal migration but rather to planet ejections and collisions with the stellar host.

We run 1200 direct integrations, 200 per panel in Figure 3. The initial mutual inclination between the inner- and outerplanet orbits, $\cos (I)$, was sampled uniformly between 0 and 1 . The initial inner- and outer-planet argument of periapsis $\omega_{1}$ and $\omega_{2}$ and the longitude of the ascending nodes $\Omega_{1}$ and $\Omega_{2}$ were chosen randomly between 0 and $2 \pi$. The outer planet initial eccentricity and semimajor axis were set equal to the observed values while the inner-planet eccentricity was initially set to a 
fixed value (0.01 and 0.2). We take the mass of the planets to be equal to the minimum mass as inferred from observations. We set $\tau=66 \mathrm{~s}$ and evolved each system for a maximum time of $10^{8} \mathrm{yr}$. In addition, we ran 10 retrograde configurations, $\cos (I)$ in the range $(-0.1,0)$, for initial conditions corresponding to HD 37605. By comparing a number of orbit-average integrations in which we adopted different values of $\tau$, we found that the results of these integrations can be rescaled using

$$
t^{\prime} \rightarrow t \times \frac{\tau}{\tau^{\prime}}
$$

so that evolving a system for $10^{8} \mathrm{yr}$ with $\tau=66 \mathrm{~s}$ would be at a good approximation equivalent to evolve the same system for $10^{10} \mathrm{yr}$ with $\tau=0.66 \mathrm{~s}$. The latter value of $\tau$ is large enough to allow the formation of $\mathrm{HJs}$ at $\lesssim 0.1$ au in $10^{10} \mathrm{yr}$ (Socrates et al. 2012a). We caution that although the scaling of Equation (13) is almost exact for stable systems, it might be an oversimplification near the region of instability given that our direct simulations cannot identify whether a system will be unstable on timescales longer than $10^{8} \mathrm{yr}$. In addition to this, the outer-planet mass could be larger than the adopted value, which will render the system even more susceptible to dynamical instabilities. It is likely therefore that in our analysis we are overestimating the number of stable systems.

Figure 3 displays the results of the direct integrations. In the top panels the inner-planet semimajor axis is initially $a_{1}(0)=1 \mathrm{au}$. In these cases most configurations are unstable, leading to planet disruptions (red curves) or ejection of one of the planets (green curves). We also checked for any collision between the two planets but did not find any. We calculate the number of "migrated" planets as those that have reached within $a_{1} \leqslant 0.5 \mathrm{au}$ at the end of the integration. As expected, for configurations that are unstable according to Equation (3) no migrating planet was formed. Three planets had $a_{1} \leqslant 0.5$ au for the initial conditions corresponding to HD 37605c, but only for retrograde configurations. This is a consequence of the backreaction torque of the inner planet on the outer orbit, which shifts the initial critical inclination at which the maximum possible $e$ is attained at $\geqslant 90^{\circ}$. Note that a more massive perturber will reduce this effect and allow the formation of WJs and HJs also for prograde configurations.

In the bottom panel of Figure 3 we set $a_{1}(0)=0.7 \mathrm{au}$. These configurations are stable according to our stability criteria, although they are near the extreme of inequality (5). At large inclinations, $\cos (I) \lesssim 0.5$, the inner-planet orbit becomes extremely eccentric, so that in most cases the planet collides with the star. This is expected since in all cases considered here the maximum eccentricity attained by the inner planet is not limited by precession due to tidal bulges, which becomes a limiting factor for the maximum $e_{1}$ only at $a_{2} / a_{1} \gtrsim 4$. Nevertheless, a few planets managed to migrate within $0.5 \mathrm{au}$ for a mutual inclination that lies initially near or above the LK critical angle, $\cos (I) \approx 0.65$. In these cases, the inner planets attain an eccentricity that is large enough to allow for efficient tidal dissipation, but, due to the mild initial inclinations, never high enough to cause the disruption of the planet. For initial inclinations smaller than $\cos (I) \approx 0.65$, the inner-planet eccentricity cannot be excited to high values so that the system is more stable, and the orbital parameters of the planets remain essentially unchanged during the evolution.

In conclusion, our direct integrations show that LK-induced migration is unlikely to occur for unstable systems, while it can lead to the formation of HJs and WJs for systems that are just below the stability boundaries, although, as shown next, these are likely to be rare.

\section{ORBIT AVERAGE TREATMENT: MIGRATING PLANETS AND THEIR ORBITAL DISTRIBUTION}

In this section we run suites of LK simulations, in which systems are initizialized with two Jupiter-mass planets at high mutual inclination. By comparing the ratio of WJs to cold Jupiters (CJs) found in the simulations to that ratio as observed, we put constraints on the fraction of observed WJs that are likely to have undergone high-e LK migration.

In our simulations we set the mass of the inner planet to $M_{1}=1 M_{\text {Jupiter }}$ and sample the mass of the outer perturber uniformly in the range $1 M_{\text {Jupiter }} \leqslant M_{2} \leqslant 17 M_{\text {Jupiter }}$. The latter values correspond approximately to the extremes of the mass distribution of the observed Jupiter companions to HJs and WJs. We assume the orbits to be prograde and sample the mutual inclination from a uniform distribution in $\cos (I)$. We take the mutual inclination in the range $50^{\circ}-90^{\circ}$. The high mutual orbital inclination is required in order for the inner planet to reach high eccentricities. We adopt a small initial eccentricity for the inner planet $e_{1}=0.1$ and take $e_{2}=0.2$ for the outer planet. The latter value is close to the median of the eccentricity distribution for the outer companion of observed HJs and WJs. The outer planet semimajor axis is sampled uniformly within $a_{2} \leqslant 8$ au, with the lower limit set such that the system satisfied the stability condition of Equation (3) in the limit $e \rightarrow 1$. We considered two values of the inner-planet semimajor axis: $a_{1}=1$ and $0.6 \mathrm{au}$. For each value of $a_{1}$ we considered three values of the time-lag constant $\tau=0.066$, 0.66 , and $66 \mathrm{~s}$, which correspond to a tidal quality factor $Q$ of $\approx 10^{6}, 10^{5}$, and $10^{3}$, respectively. Thus, we evolved six sets of initial conditions. For each set we performed a total of 1000 random realizations, integrating them up to a final integration time of 10 Gyr.

In addition to the secular integrations described above, we ran three sets of initial conditions (corresponding to the three values of $\tau$ ) where we used a Rayleigh distribution for both $e_{1}$ and $e_{2}$ with a mean eccentricity of 0.175 (Moorhead et al. 2011), and this time sampling $a_{2}$ such that the system satisfied the stability condition of Equation (5). In the latter simulations (models $\mathrm{C}$ in Table 2), the planets can have initially a lower $a_{2} / a_{1}$ ratio, which favors the formation of WJs as discussed in Section 2. As a consequence of this, such models are expected to produce more WJs than if we were to select the initial conditions based on inequality (3); however, we caution that they also contain more systems that are near the orbit crossing when $e_{1} \approx 1$ and for which the orbit-averaged treatment we use is less accurate.

Table 2 gives the initial setup of the numerical integrations and summarizes the main results of our simulations, giving the fraction of systems that lead to the specified outcomes. In order to take into account the fact that we are only simulating systems with mutual inclinations larger than $50^{\circ}$ we reduced the number of forming HJs and WJs (and disrupted) by $\cos 50^{\circ}$, and then used that number in the fraction. We find that a high-e LK migration scenario is more efficient at producing HJs than WJs. The fraction of systems that result in the formation of WJs is $\approx 1 \%$ of the total and it is roughly constant, having little variation with the initial conditions and tidal dissipation strength. The fact that the number of WJs is not very sensitive 
Table 2

Results of Secular Integrations

\begin{tabular}{|c|c|c|c|c|c|c|c|c|c|}
\hline Model & $\begin{array}{l}\text { Stability } \\
\text { Criterion }\end{array}$ & $\begin{array}{l}a_{1}(0) \\
(\mathrm{au})\end{array}$ & $e_{1}(0)$ & $e_{2}(0)$ & $\begin{array}{c}\tau \\
(\mathrm{s})\end{array}$ & $\begin{array}{l}\mathrm{HJs} \\
(\%)\end{array}$ & $\begin{array}{l}\text { WJs } \\
(\%)\end{array}$ & $\begin{array}{c}\text { Nonmigrating } \\
(\%)\end{array}$ & $\begin{array}{c}\text { Disrupted } \\
(\%)\end{array}$ \\
\hline$\overline{\mathrm{A} 1}$ & P15 & $1 \mathrm{au}$ & 0.1 & 0.2 & 0.066 & 0.77 & 1.02 & 70.7 & 27.5 \\
\hline A 2 & $\ldots$ & $1 \mathrm{au}$ & 0.1 & 0.2 & 0.66 & 1.22 & 0.90 & 64.4 & 33.5 \\
\hline B1 & $\ldots$ & $0.6 \mathrm{au}$ & 0.1 & 0.2 & 0.066 & 1.73 & 0.70 & 66.4 & 31.2 \\
\hline B2 & $\ldots$ & $0.6 \mathrm{au}$ & 0.1 & 0.2 & 0.66 & 4.41 & 0.51 & 65.1 & 29.9 \\
\hline B3 & $\ldots$ & $0.6 \mathrm{au}$ & 0.1 & 0.2 & 66 & 21.8 & 0.83 & 56.9 & 20.5 \\
\hline $\mathrm{C} 3$ & $\ldots$ & $0.6 \mathrm{au}$ & Rayleigh & Rayleigh & 66 & 19.7 & 2.05 & 60.4 & 17.9 \\
\hline $\mathrm{C} 1-\mathrm{c}$ & $\ldots$ & $0.6 \mathrm{au}$ & Rayleigh & Rayleigh & 0.066 & 1.86 & 0.58 & 79.0 & 18.6 \\
\hline $\mathrm{C} 2-\mathrm{c}$ & $\ldots$ & $0.6 \mathrm{au}$ & Rayleigh & Rayleigh & 0.66 & 4.41 & 0.96 & 74.3 & 20.3 \\
\hline C3-c & $\ldots$ & $0.6 \mathrm{au}$ & Rayleigh & Rayleigh & 66 & 16.1 & 1.73 & 67.2 & 15.0 \\
\hline
\end{tabular}

Note. We define here as HJs those planets that by the end of the simulation have migrated within $0.1 \mathrm{au}$; WJs are defined as those planets that are in the semimajor axis range 0.1-0.9 au (for $a_{1}(0)=1 \mathrm{au}$ ) or $0.1-0.5 \mathrm{au}$ (for $a_{1}(0)=0.6 \mathrm{au}$ ). Nonmigrating planets have final semimajor axis $a_{1} \geqslant 0.9 \mathrm{au}\left(\right.$ for $a_{1}(0)=1 \mathrm{au}$ ) or $a_{1} \geqslant 0.5 \mathrm{au}$ (for $a_{1}(0)=0.6 \mathrm{au}$ ); "disrupted" systems are those in which the inner planet had crossed its Roche limit. In the first models we ran the integrations up to a maximum time of $10 \mathrm{Gyr}$. In the last three models (C1-c, C2-c, C3-c) the final integration time was chosen randomly between 0 and 10 Gyr. The latter choice is to simulate a scenario in which the planets have formed continuously over the past $10 \mathrm{Gyr}$. In order to account for the fact that we have only selected systems with an inclination $50^{\circ}$, we have reduced the number of forming HJs and WJs (and disrupted) by $\cos 50^{\circ}$ and then used that number in the fraction.

to $\tau$ is because increasing $\tau$ will result in a larger number of planets migrating inside the period valley, but at the same time also in a larger number of planets leaving it. According to our results, if WJs are migrating planets, then for a homogeneous sample of planets we we would expect that planets with detected outer companions would more often be HJs than WJs. This expectation appears to be at odds with what is observed. Restricting our analysis to the subset of known extrasolar planets discovered by radial velocity surveys, only two of the Jupiter-mass planets $\left(M_{1} \sin i \geqslant 0.5 M_{\text {Jupiter }}\right)$ at $\leqslant 0.1$ au have a detected outer companion within $\lesssim 5$ au distance from their host star, while 20 Jupiter-mass planets at 0.1 au $\leqslant a_{1} \leqslant 1$ au do. This suggests that HJs and WJs belong to two distinct populations of planets that likely originated through different processes.

Figure 4 shows the properties of outer companions to the observed HJs (black filled circles) and WJs (black stars). The black and red curves show the value of $a_{2}$ satisfying the condition given by Equations (3) and (5), respectively. Below these lines WJs are unlikely to form as the system is unstable to either collision or ejection of one of the planets. As done previously in Section 2, we computed the stability limit from Equation (3) by taking the relevant limit $e_{1} \rightarrow 1$. In Figure 4 the relative position of the black filled circles to the stability lines shows that only 4 of the 24 observed systems would be dynamically stable if the inner planet had $a_{1}=1 \mathrm{au}$, and 14 of them would be on intersecting orbits. Figure 4 also displays the results from models $\mathrm{A} 1$ and $\mathrm{A} 3$ of Table 2, showing the systems that form HJs (red filled circles) and those that form WJs (red stars), the latter being defined as those systems in which the innermost planet had experienced significant tidal dissipation and migrated inside the semimajor-axis range $0.1-0.9 \mathrm{au}$. From this figure we see again that the number of migrated planets that have formed an HJ increases significantly when increasing the time-lag factor $\tau$, while the number of migrating planets in the period valley (red stars) increases but only slightly. Accordingly, the number of HJs formed in our models is typically equal to or larger than the number of migrating WJs.
In the top panel of Figure 5 we show the period distribution of migrating planets and compare this to the (intrinsic) observed distribution from Santerne et al. (2016). Such comparison shows that the semimajor-axis distribution of the planets in our simulations does not provide a good match to the observed period distribution of gas giants in the period valley. Given the observed number of Jupiters at $P \gtrsim 100$ days, the models underpredict the number of migrating planets below this period by at least one order of magnitude. We conclude that a high-e migration mechanism can be responsible for less than $10 \%$ of all gas giants with orbital periods in the range of 10-100 days.

We note that our simulations are restricted to systems with inner planets initially at $\leqslant 1 \mathrm{au}$, accompanied by an outer perturber within $\leqslant 8$ au. A significant contribution to the WJ population from planets migrating from $\gg 1$ au and having companions at $\gg 8$ au is unlikely. In fact, as also noted in Section 2, WJs cannot form for Jupiter mass perturbers if $a_{2}$ is larger than a few au.

Finally, we note that, similar to previous studies (e.g., Anderson et al. 2016), our model distributions do not match the period distribution of $\mathrm{HJS}_{\mathrm{S}}(<10$ days), producing too many planets at short orbital periods ( $\sim 1$ day). Such a discrepancy might depend on the choice we made for various parameters, most importantly on the adopted value of the planet radius, which determines the final semimajor axis of the $\mathrm{HJ}(\mathrm{Wu} \&$ Lithwick 2011).

A secular migration model for WJs fails at explaining some additional features of the observed orbital distribution. First, in only 3 of the 3000 models that were started at $a_{1}(0)=1$ au was the inner planet found in the region $0.1-0.5$ au by the end of the simulation. This is in contrast with the abundance of observed Jovian planets at these radii and casts further doubts on a possible migratory origin from $\gtrsim 1$ au for these systems. If the planets started with a smaller semimajor axis $a_{1}(0)=0.6 \mathrm{au}$, as shown in Figure 5, then for each Jupiter in the radial range 0.1-0.5 au our models produce at least 10 more (mostly nonmigrating) Jupiters within the range $0.5-1$ au. Contrary to 

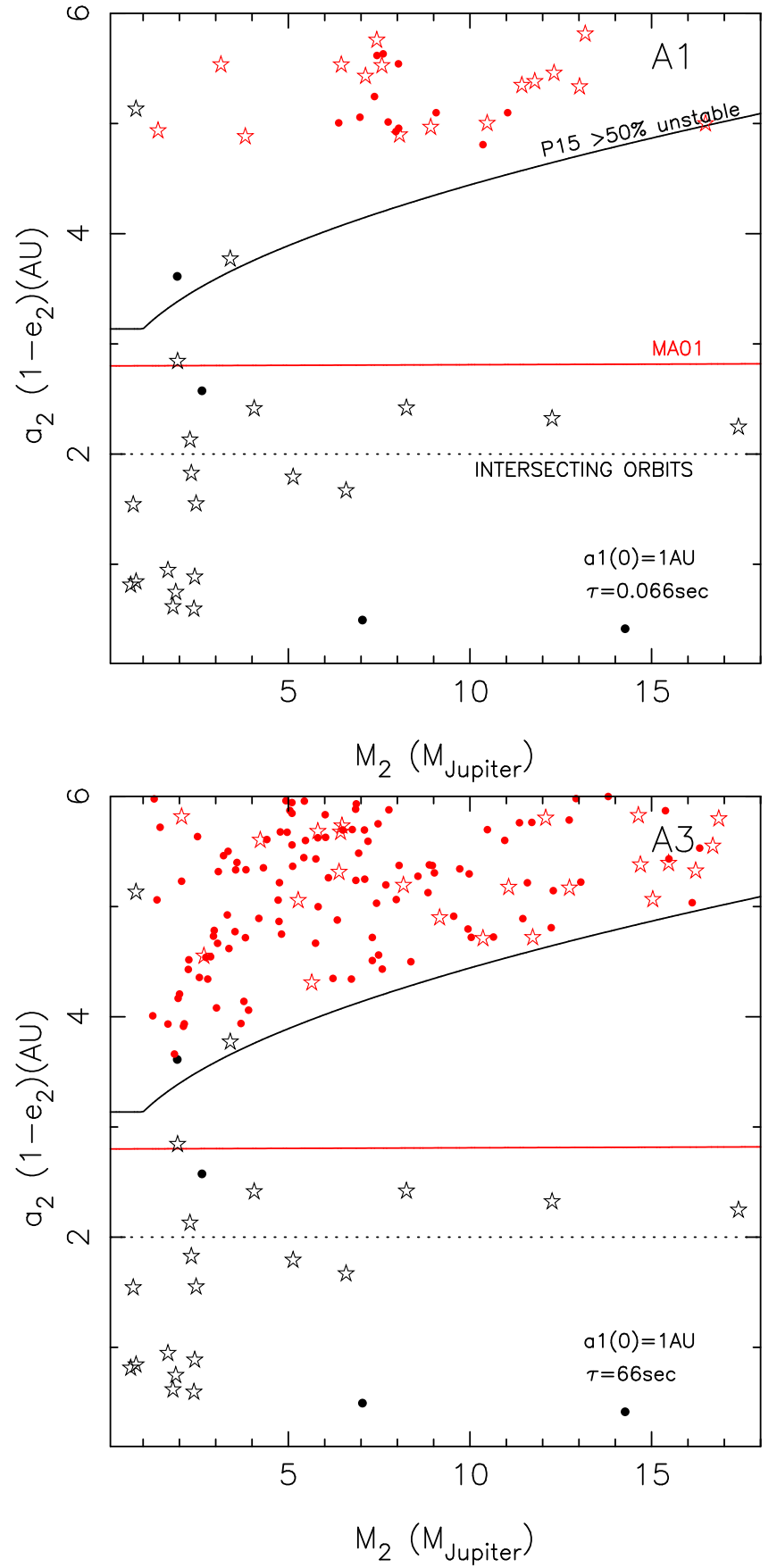

Figure 4. Solid lines show regions of stability. Any proto-HJ/WJ with $a_{1}=1 \mathrm{au}, e_{1}=1$, and $M \sin I \geqslant 0.5 M_{\text {Jupiter }}$ would be unstable according to Equation (5) if its external companion lay below the horizontal red line (labeled MA01). The other solid line corresponds to Equation (3). Below the dashed line the two planets would be on intersecting orbits. Simulation results are shown for models A1 and A3 (see Table 2). Red stars indicate WJs, i.e., those systems in which the inner planet has experienced significant tidal dissipation and migrated within 0.9 au but has not evolved inside 0.1 au. Red filled circles are systems that have formed an $\mathrm{HJ}\left(a_{1}<0.1 \mathrm{au}\right)$ at the end of the integration. Black symbols correspond to the observed systems of Table 1. Black stars are WJs $\left(a_{1}=0.1-1 \mathrm{au}\right)$; black filled circles are HJs $\left(a_{1}<0.1 \mathrm{au}\right)$.

this, observations yield roughly the same number of giant planets in these two ranges of semimajor axes.

The bottom panel of Figure 5 shows the final $a$ versus $e$ distribution of the simulated systems compared to the observed distribution. This plot shows an additional important feature of
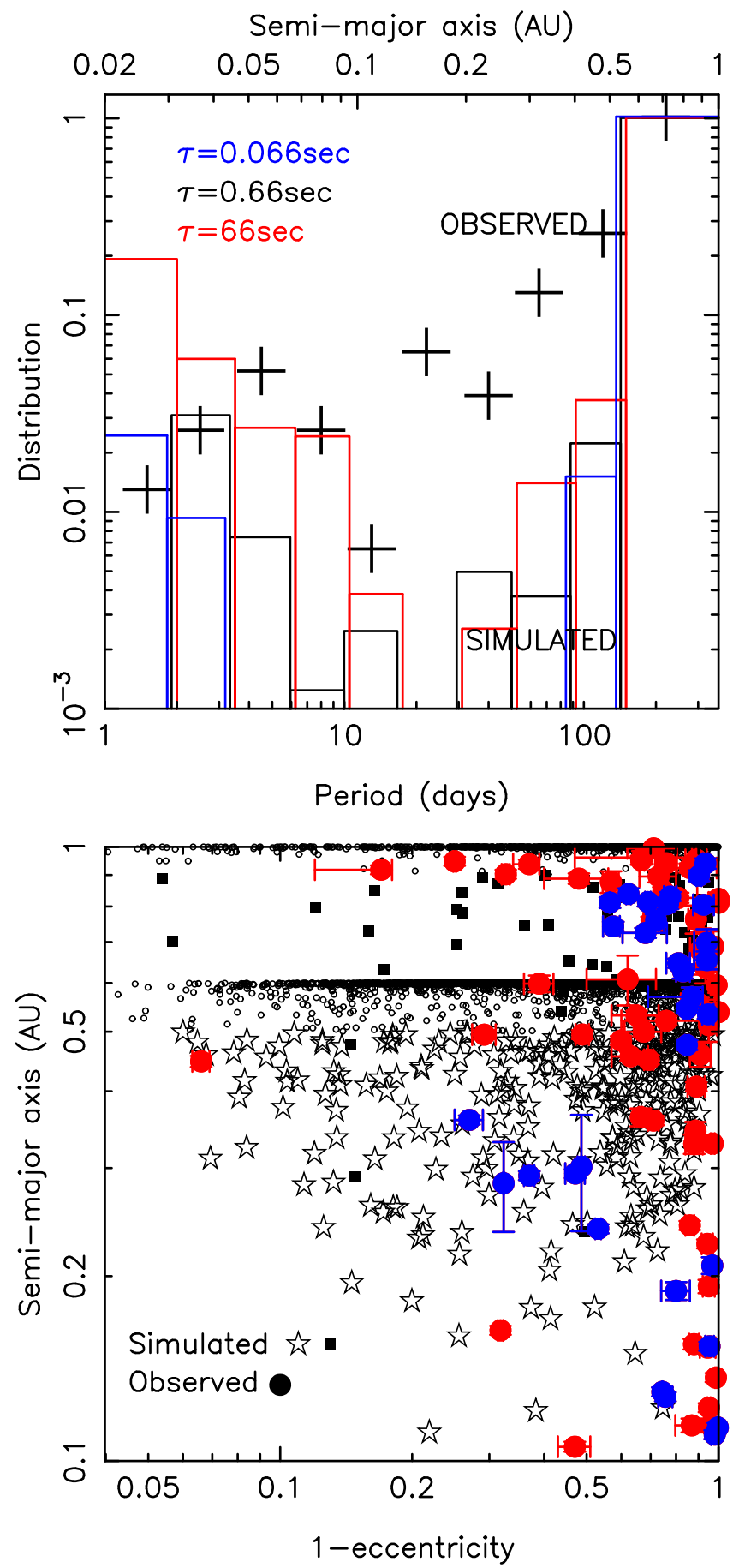

Figure 5. Top panel: semimajor-axis distribution of the planets from our simulations (histogram) compared to the observed distribution from Santerne et al. (2016) (plus signs). The simulated distributions are from the C1-c, C2-c, and C3-c models that have $a_{1}(0)=0.6$ au (see Table 2). Other models that have $a_{1}(0)=0.6$ au were found to produce similar distributions. The observed and simulated distributions have been normalized such as to have the same value at periods $P \gtrsim 200$ days. Note how all of our models greatly underestimate the number of Jupiters in the WJ zone. Bottom panel: $a$ vs. $e$ distribution of the inner planets for systems that were evolved forward in time using the secular equations of motion. Small open circles are systems that have not experienced significant inward migration. Filled squares (stars) correspond to planets with $a_{1}(0)=1 \mathrm{au}\left(a_{1}(0)=0.6 \mathrm{au}\right)$ and that migrated within 0.9 au $(0.5 \mathrm{au})$ by the end of the simulation. Note that models with $a_{1}(0)=1 \mathrm{au}$ produce almost no WJs inside 0.5 au. These distributions are compared to observational data, which are represented by the filled circles. Data points corresponding to Jupiters with a known Jupiter companion are in blue. In the bottom panel data are from exoplanets.org. 


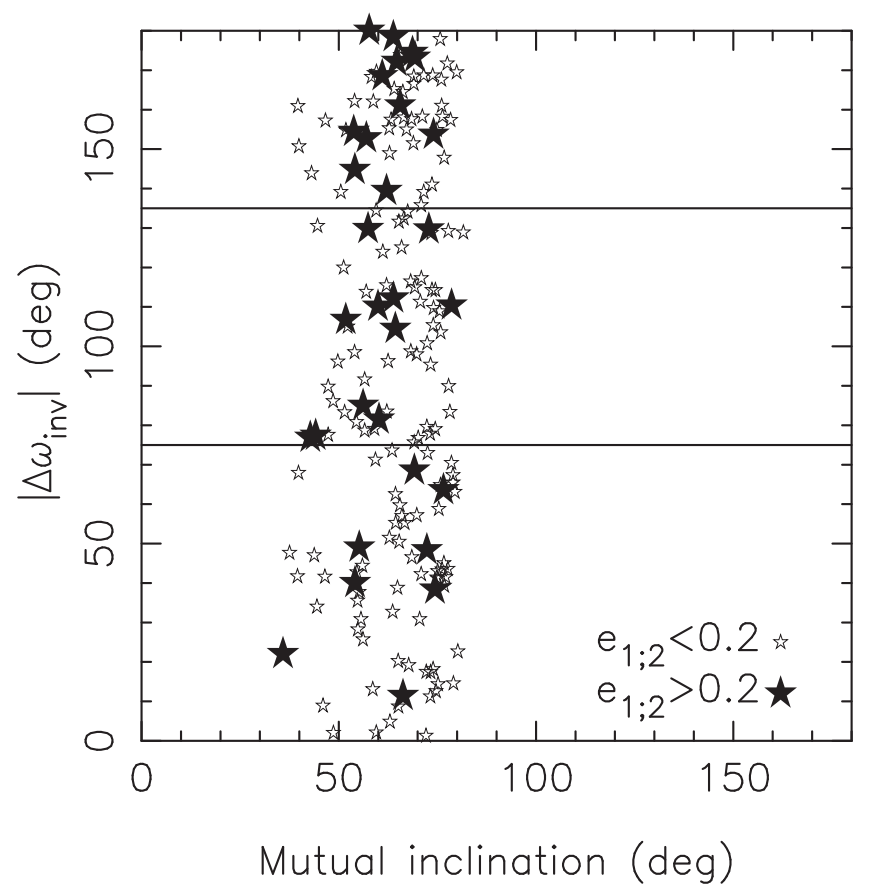

Figure 6. Difference in apsidal longitudes, $\Delta \omega_{\text {inv }}=\omega_{1}-\omega_{2}$, as a function of mutual inclination for the WJs formed in our secular integrations. Filled stars are the subset of systems comprising an eccentric WJ with an eccentric outer companion. $e_{1}$ and $e_{2}$ are the final value of the eccentricity of inner and outer planets, i.e., those at the time at which the apsidal longitudes were computed.

the simulated distribution that is difficult to reconcile with observations: the lack of low-e WJs at $a_{1} \lesssim 0.3 \mathrm{au}$. In fact, none of our models produced a WJ with $e \lesssim 0.3$ at these radii, while $\sim 80 \%$ of known planets with semimajor axis $0.25 \leqslant a_{1} \leqslant 0.1$ have eccentricities $\lesssim 0.3$. Evidently giant planets observed in this region of parameter space are unlikely to have formed through secular migration induced by an outer perturber planet. The latter point is further discussed in the next section.

Dawson \& Chiang (2014) showed that eccentric WJs with eccentric outer giant companions have apsidal separations that cluster near $90^{\circ}$. Dawson \& Chiang (2014) interpreted this as a signature of mutual inclinations being between $35^{\circ}$ and $65^{\circ}$, favoring LK migration as the mechanism for the formation of the inner planets. In Figure 6 we show the distribution of the apsidal misalignment, $\Delta \omega_{\text {inv }}=\omega_{1}-\omega_{2}$, as a function of mutual inclination for the migrating WJs in our secular integrations. Interestingly, our simulated planets do not show any significant clustering around $\Delta \omega_{\text {inv }}=90^{\circ}$. The WJs formed in our models have mutual inclinations in the range $40^{\circ}-80^{\circ}$, but their $\Delta \omega_{\text {inv }}$ appear to be uniformly distributed. These results suggest some other process, other than LK oscillations, as being responsible for the near orthogonality of apsides exhibited by the observed WJs.

\subsection{Evolution toward Low Semimajor Axes}

As shown in Figure 5, a high-e LK migration scenario for WJs fails at producing systems with a low value of $a$ and $e$, which are indeed quite numerous among the observed systems. In fact, none of our simulations produced a system in the region where, for example, HD 38529b and HD 13908b are observed (0.1-0.3 au), disfavoring an LK-induced migration scenario for the formation of these planets. As discussed below, the reason for this is the reduced range of eccentricity oscillations due to Schwarzschild precession as the planet semimajor axis shrinks due to tidal dissipation.

At the quadrupole-order level and in the test particle limit the quantity

$$
H=\ell^{2}+\sin ^{2} I\left(\ell^{2}+5 e_{1}^{2} \sin ^{2} \omega\right)-\frac{k}{\ell},
$$

with $\ell=\sqrt{1-e_{1}^{2}}$, is an integral of motion as it differs from the system Hamiltonian only by a constant (e.g., Merritt 2013). The third term on the right-hand side of Equation (14) represents the extra Schwarzschild precession term, where

$$
k=8 \frac{M_{\star}}{M_{2}} \frac{r_{g} a_{2}^{3}}{a_{1}^{4}}\left(1-e_{2}^{2}\right)^{3 / 2},
$$

and $r_{g}=G M_{\star} / c^{2}$.

From the conservation of $H$ and $\ell_{z}=\ell \cos I$ we can derive a relation between the maximum $\left(\ell_{+}\right)$and minimum $\left(\ell_{-}\right)$angular momentum attained during an LK oscillation for a given value of $\ell_{\mathrm{z}}$. When the argument of periapsis $\omega$ librates around $\pi / 2$, the maximum and minimum values of $\ell$ are related through the equation

$$
\ell_{+}^{2} \ell_{-}^{2}=\frac{5}{3} \ell_{z}^{2}+\frac{k}{3}\left(\frac{\ell_{+}-\ell_{-}}{\ell_{+}^{2}-\ell_{-}^{2}}\right) \ell_{+} \ell_{-} .
$$

As the planet semimajor axis decreases due to tidal dissipation, relativistic precession will increase the portion of parameter space available for circulation at the expense of libration, gradually pushing an initially librating orbit toward the separatrix at which $\ell_{+}=1$, and finally onto a circulating orbit (see also Blaes et al. 2002; Anderson et al. 2016, for a similar analysis).

The distance at which the fixed point no longer exists is found by setting $\omega=\pi / 2$ and $\dot{\omega}=0$, which yields

$$
\begin{aligned}
\tilde{a}= & {\left[4 \frac{M_{\star}}{M_{2}} \frac{r_{g} a_{2}^{3}\left(1-e_{2}^{2}\right)^{3 / 2}}{3-5 \ell_{z}^{2}}\right]^{1 / 4} \approx 0.2\left(\frac{M_{\star}}{M_{\odot}}\right)^{1 / 2} } \\
& \times\left(\frac{M_{2}}{M_{\text {Jupiter }}}\right)^{-1 / 4}\left(\frac{a_{2} \sqrt{1-e_{2}^{2}}}{3 \mathrm{au}}\right)^{3 / 4}\left(2 \frac{a_{1}}{a_{1}-a_{\mathrm{cr}}}\right)^{1 / 4} \mathrm{au},
\end{aligned}
$$

where we have used the fact that $\ell_{z} \approx \sqrt{3 / 5} \ell_{-}$(from $\ell_{z}=\cos (I) \ell$ and $\cos (I) \approx \sqrt{3 / 5}$ at $\left.\ell_{-}\right)$and set $\ell_{-}=a_{\mathrm{cr}} / a_{1}$ as required for efficient tidal dissipation to occur. Below $\tilde{a}$ librating solutions no longer exist.

After $\omega$ starts circulating, $\omega=\pi / 2$ at $\ell=\ell_{-}$and $\omega=0$ at $\ell=\ell_{+}$, which leads to the relation

$$
\ell_{+}^{2}=\frac{5}{2}\left(1+\ell_{z}^{2}-\frac{3}{5} \ell_{-}^{2}-\frac{\ell_{z}^{2}}{\ell_{-}^{2}}\right)+\frac{k}{2}\left(\frac{1}{\ell_{+}}-\frac{1}{\ell_{-}}\right) .
$$

According to Equation (18) and for $a_{1}<\tilde{a}, \ell_{+}$must become smaller as $a_{1}$ decreases, thereby pushing the planet away from the region of small $a_{1}$ and $e_{1}$.

A good approximation to Equation (18) can be obtained by noting that $\ell_{z} \approx \sqrt{3 / 5} \ell_{-}$and $k / \ell_{+} \approx k$ (from $\left.\ell_{+} \approx 1\right)$, which 

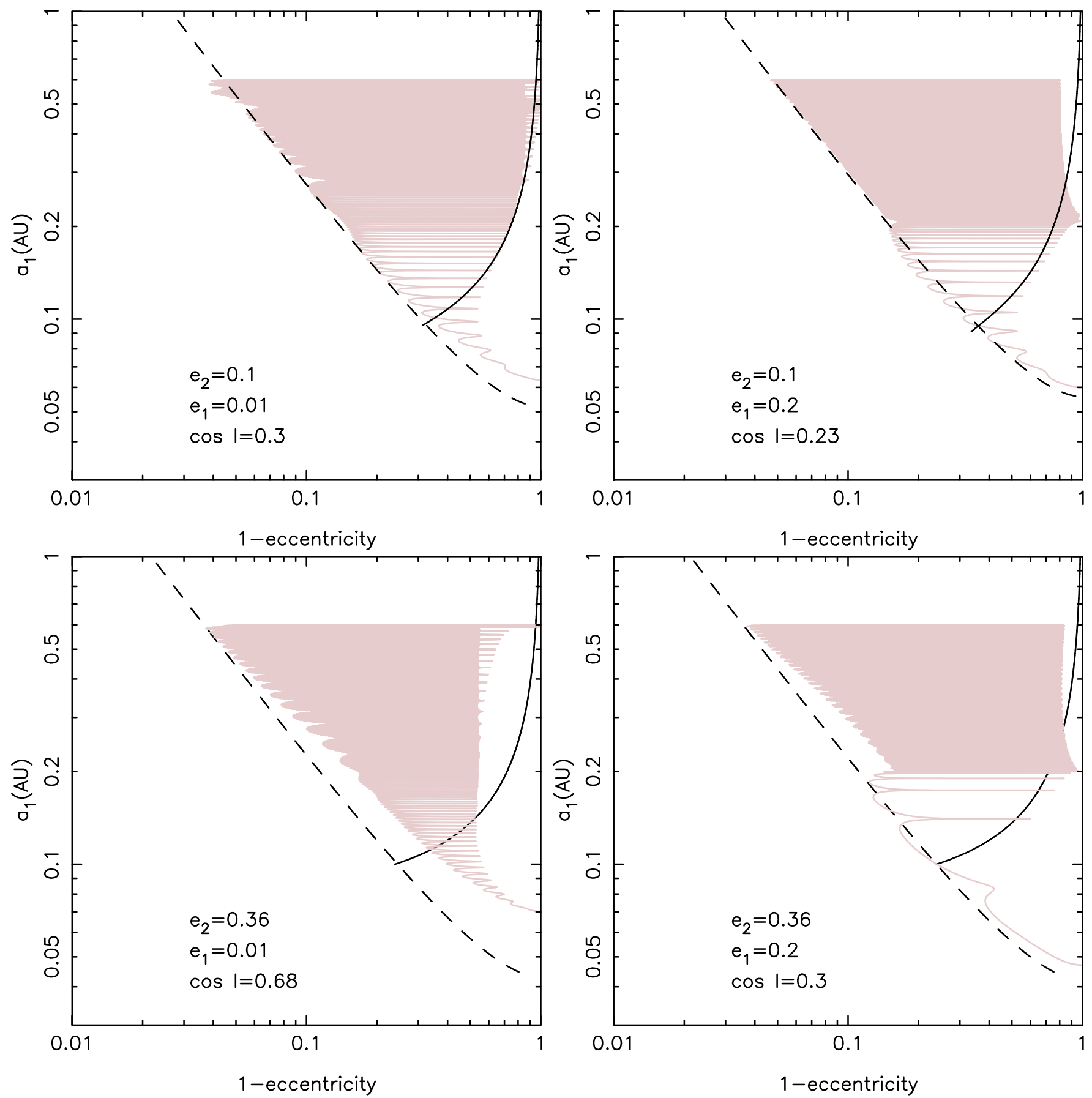

Figure 7. Orbital evolution of migrating planets obtained with the secular equations of motion. The solid lines give the minimum eccentricity attained during the LK oscillations as predicted by Equation (19); dashed lines are lines of constant angular momentum that give the value of $\ell_{-}$we have used to compute the solid line. In the bottom panels the eccentricity of the outer planet is $e_{2}=0.36$, which causes the octupole-order terms to become important to the evolution. In all panels the innerplanet argument of periapsis was set to $\omega_{1}=\pi / 2$ and the mass of the outer planet was $17 M_{\text {Jupiter }} ; \omega_{2}$ was set equal to (in radians) 3 (top left), 0.013 (top right and bottom left), and 2.6 (bottom right).

leads to the simpler relation

$$
\ell_{+}^{2} \approx 1+\frac{k}{2}\left(1-\frac{1}{\ell_{-}}\right)
$$

for circulating orbits. Although quite simplified and reasonable only for an orbit close to the separatrix, Equation (19) was found to reproduce the results of numerical simulations fairly well. A few example systems are shown in Figure 7. The dashed curve in the panels that demarcate the $\ell_{-}$envelope is a curve of constant angular momentum: $\sqrt{a_{1}} \ell . \ell_{-}$tracks this curve because tidal dissipation occurs mostly at $\ell=\ell_{-}$. In the top left panel the inner-planet argument of periapsis is initially circulating and the $\ell_{+}$value steadily decreases with time. In the top right panel instead $\omega$ is initially librating. From Equation (16) we see that the inclusion of the extra Schwarzschild precession term will tend to increase $\ell_{+}$as the orbit shrinks. Accordingly, from Figure 7 we see that as the orbit decays, $\ell_{+}$increases until it crosses the separatrix at 


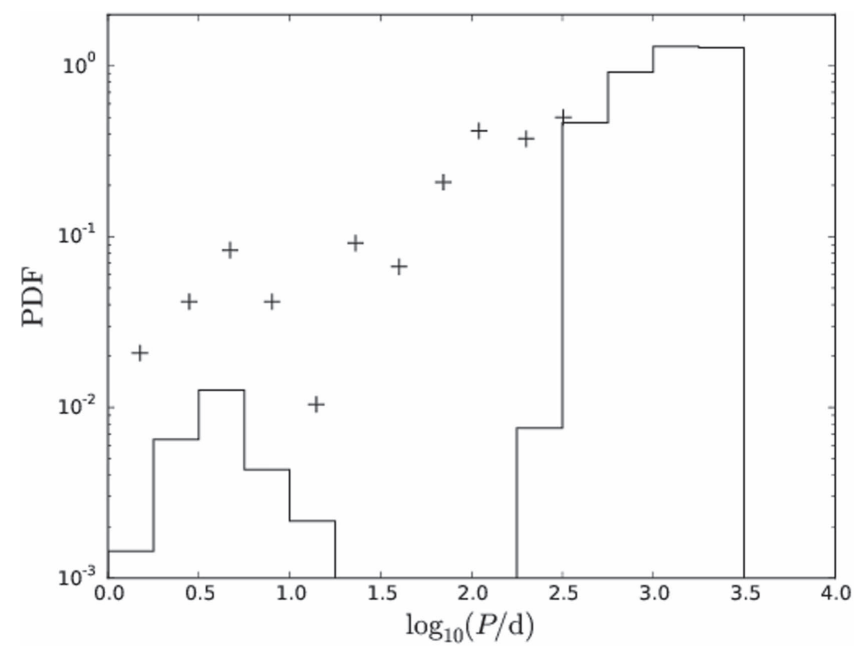

Figure 8. Period distributions from secular calculations of high-e migrations in multiplanet systems, with three to five planets (Hamers et al. 2016). Plus signs show the observational data from Santerne et al. (2016), normalized to match the simulated distribution at $\log _{10}(P / d) \approx 2.6$.

$a_{1} \approx 0.2 \mathrm{au}$, where $\ell_{+} \approx 1$. Then the $\ell_{+}$envelope is set by the separatrix, as modified by Schwarzschild precession, and it is approximately equal to the value given by Equation (19) after $\omega$ starts circulating.

\subsubsection{Effect of Octupole-order Terms}

If the orbit of the outer planet has a substantial eccentricity (typically $\gtrsim 0.1$ ), then the octupole-order terms can cause the evolution of the inner-planet orbit to deviate significantly from the simple model depicted above. However, the distribution shown in Figure 5, which was obtained with the octupole secular code, suggests that even when higher-order terms are included, the innermost planet orbit stays away from the region $a_{1} \lesssim 0.3 \mathrm{au}, e_{1} \lesssim 0.3$, for $a_{2} \geqslant 0.6 \mathrm{au}$.

The octupole-order terms have two main effects: (i) the higheccentricity part of the envelope can deviate significantly from a line of constant angular momentum (e.g., bottom panels of Figure 7); (ii) if the inner-planet argument of periapsis is initially librating, as the semimajor axis decreases due to tidal friction, the orbit eventually crosses the LK separatrix, where dynamical chaos can drive the orbital eccentricity to very high values. When this happens, the planet orbit will tend to "freeze" at higher values of $e$ so that an HJ will promptly form (see the bottom right panel of Figure 7).

\subsection{Multiplanet Systems and Secular Chaos}

As mentioned before, another method to excite eccentricities is via secular chaos in multiplanet systems (Wu \& Lithwick 2011). Here we show that the conclusions drawn in this section likely apply to these types of systems as well.

In systems that host more than two giant planets, the planets need not be close companions and need not be initially highly eccentric and/or inclined to excite the innermost planet eccentricity to high values and potentially produce a WJ (e.g., Hamers et al. 2015). However, population synthesis studies with plausible assumptions (Hamers et al. 2016) find that almost no WJs are produced, whereas HJs are produced in more significant numbers (up to a few percent).
In Figure 8 we show the period distribution of a large set of multiplanet-system integrations based on the orbit-averaged code described in Hamers \& Portegies Zwart (2016). In these secular simulations, the number of planets was chosen to be between three and five, and the semimajor axes were sampled linearly in the ranges of 1-4 au, 6-10 au, 15-30 au, 45-50 au, and 60-100 au for the three to five planets, respectively. The stellar mass was set to $1 M_{\odot}$, and the planetary masses were sampled randomly between $0.5 M_{\text {Jupiter }}$ and $5 M_{\text {Jupiter }}$. The arguments of periapsis and longitudes of the ascending nodes were sampled randomly. The apsidal motion constant was set to 0.28 . The time-lag constant was set to $\tau=66 \mathrm{~s}$, and the innermost planet radius was set to either $1 R_{\text {Jupiter }}$ or $1.5 R_{\text {Jupiter }}$. The inclinations and eccentricities (in units of radians) were sampled from a Rayleigh distribution with an rms width of either $10^{\circ}$ or $15^{\circ}(\approx 0.18$ or $\approx 0.35$ radians $)$.

In total we integrated 10,000 systems up to a maximum integration time of $10 \mathrm{Gyr}$. In our analysis we rejected all systems in which the planet orbits crossed during the integration or in which the inner planet collided with the star. We also tried different values of $\tau(0.66$ and $0.066 \mathrm{~s})$ but found this not to affect our conclusion: similar to the results of the two-planet system integrations described above, and in stark contrast with the observations, a small number of WJs are produced compared to HJs. Indeed, our multiplanet simulations produce essentially no Jupiter in the period valley, as can be seen in Figure 8. We conclude that these models also greatly underpredict the number of giant planets observed in the period valley.

\section{ECCENTRICITY DISTRIBUTION}

Figure 9 compares the eccentricity distribution of our simulated systems to the distribution of observed WJs. The simulated models yield an eccentricity distribution for the migrating planets that is nearly uniform $(N(<e) \sim e)$. A comparison of these two populations shows that our migration models produce too many highly eccentric WJs to be consistent with observations. The oscillations required to produce inward migration result in more eccentric planets than observed, so that the simulated distribution for the migrating population is inconsistent with the observed eccentricity distribution (see also Dawson et al. 2015; Frewen \& Hansen 2016). The discrepancy of the migration model with observations is therefore due to the significant fraction of migrating WJs with high eccentricity, while only a few observed WJs are on higheccentricity orbits (see Figure 5). In fact, as also noted above, a large number of WJs have an eccentricity that is close to zero.

In Figure 9 we show the model eccentricity distributions starting from different initial conditions (models A3, B3, and C3-c in Table 2). These models all produce a similar final eccentricity distribution, demonstrating that our conclusions are quite robust and do not depend significantly on the choice we made for the initial conditions. Using the KolmogorovSmirnov (K-S) test gives $p$-values in the range of $10^{-3}-10^{-4}$, indicating that the synthetic and observed $e$-distributions are unlikely to be drawn from the same population.

The dashed curves in the top panel of Figure 5 give the eccentricity distribution of a planet undergoing LK oscillations that we computed using the secular equations of motion at the quadrupole (upper curve) and octupole (lower curve) level of approximation. The planet was placed at $a_{1}(0)=0.5$ au with a negligible initial eccentricity $\left(e_{1}(0)=0.01\right)$. The outer planet 

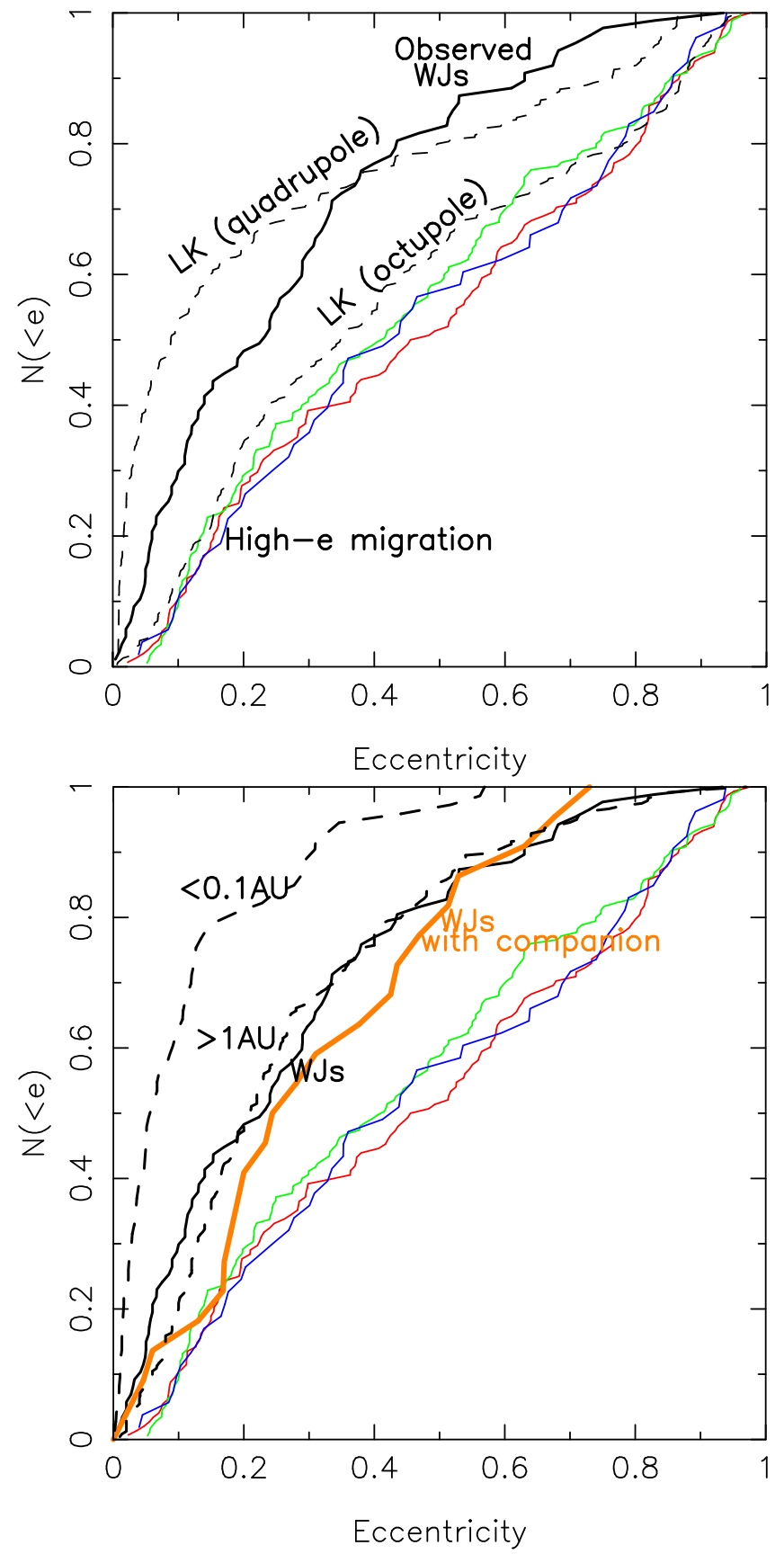

Figure 9. Cumulative eccentricity distribution of migrating planets from our two-planet simulations (green, red, and blue lines) compared to the observed eccentricity distribution of WJs (black solid lines). All the high-e migration models produce an eccentricity distribution that is significantly different from the observed distribution. In the top panel the dashed curves give the eccentricity distribution of a planet undergoing LK oscillations computed using the secular equations of motion and at the quadrupole (upper curve) and octupole (lower curve) level of approximation. In the bottom panel the black dashed curves are the eccentricity distributions of observed Jupiters in the indicated range of semimajor axes. The orange solid line is for the subsample of WJs with one outer companion. The model predictions do not take into account eccentricity-dependent selection effects, which might become important at $e \gtrsim 0.8$.

had a mass of $5 M_{\text {Jupiter }}$ and was placed at $a_{2}=6$ au with $e_{2}(0)=0.2$. The mutual inclination was set to $70^{\circ}$. At the quadrupole level of approximation we can simply derive the eccentricity distribution as $d N / d e=d t / d e \sim 1 / e_{1}$ so that $N \sim \ln e-$ this follows from the fact that in a mixed ensemble the number of planets $\Delta N$ in the interval $e \sim e+\Delta e$ is proportional to $\Delta t$. As expected, this form matches quite well the eccentricity distribution given by the upper dashed curve in Figure 5, but does not provide a good mach to either the observed eccentricity distribution or the model distribution. The distribution of the migrating Jupiters in our two-planet simulations is instead similar to that of a population of planets undergoing LK oscillations with a non-negligible contribution from the octupole potential (lower dashed curve). We conclude that the dynamical evolution of the planets in our simulations is significantly affected by the octupole-order terms. The main effect of the octupole-order terms is to skew the eccentricity distribution of WJs toward higher values.

In the bottom panel of Figure 9 we compare the eccentricity distribution of planets within the range of semimajor axes: $0.1-1 \mathrm{au}, \leqslant 0.1 \mathrm{au}$, and $\geqslant 1 \mathrm{au}$. Interestingly, we find that the eccentricity distribution of giant planets in the radial range 0.1-1 au is consistent with that of planets at radii larger than 1 au. A K-S test on these distributions gave a $p$-value of 0.31 , implying that the two samples are consistent with being taken from the same distribution. For comparison, the same test between the distribution of planets with $a>1$ au and giant planets at $a<0.1$ au gave a $p$-value of $\sim 10^{-5}$. Hence, the observed $e$-distribution provides no evidence for differences in the eccentricity distribution of WJs and Jupiters outside $1 \mathrm{au}$, which are expected on the basis of theoretical models. In addition, comparing the eccentricity distribution of all WJs with that of only WJs with one outer companion (orange curve) shows that these two distributions are not significantly different from each other. All these results point either to disk migration (Goldreich \& Tremaine 1980) or to in situ formation (Batygin et al. 2016; Boley et al. 2016) for the origin of WJs rather than secular migration processes such as the LK mechanism (Dawson \& Chiang 2014) or secular chaos (Wu \& Lithwick 2011).

\section{DISCUSSIONS AND CONCLUSIONS}

We have considered the population of giant planets in the period valley. These planets, often referred to as WJs, have orbital periods larger than 10 days but are interior to the peak of giant planet frequency observed at $\approx 1$ au (Santerne et al. 2016). It has been argued that such planets might not be able to form in situ. In a widely discussed model for the formation of these planets, large-amplitude eccentricity oscillations induced by an external perturber are followed by efficient tidal dissipation, which causes the orbit of the inner planet to shrink during close passages by the host star (e.g., Dawson \& Chiang 2014; Dong et al. 2014; Frewen \& Hansen 2016).

Before summarizing our results, we briefly address the importance of selection effects. In fact, when comparing the predictions of our models to observations, we have so far neglected the fact that observations might be biased against, for example, orbits with high eccentricity and/or large semimajor axis. This might affect the inference of the intrinsic orbital distribution of the observed planets (e.g., Socrates et al. 2012b).

We have considered mostly radial velocity data from the exoplanets.org database. Radial velocity data might be biased against the detection of longer-period planets, although this effect is likely to be small for the semimajor-axis range considered here, $a<1$ au (Cumming 2004). More important might be the bias against the detection of eccentric planets, which would affect the distributions of Figure 9. In fact, the 
sparse sampling of an orbit with a high eccentricity can miss the reflex velocity signal near periapsis, leading to nondetection of planets that would be detected at the same semimajor axis and lower eccentricity (Cumming 2004). We believe, however, that these effects should be relatively small since our sample is restricted to giant planets with relatively large masses $\left(M \sin I>0.5 M_{\text {Jupiter }}\right)$ and small semimajor axes $(<1 \mathrm{au})$, which should be relatively easy to detect. We note also that other studies that have taken into account such selection effects have reported results similar to ours, pointing out the excess of highly eccentric WJs predicted by high- $e$ scenarios compared to the observed distribution (Dawson et al. 2015; Frewen \& Hansen 2016).

We also note that we have limited the parameter space of our simulations by keeping the perturber mass within $17 M_{\text {Jupiter }}$. However, the WJs for which there is no observational evidence for a Jupiter companion might be migrating due to interactions with a distant stellar companion. Petrovich (2015a) and Anderson et al. (2016) conducted octupole-level population synthesis studies of giant planets migrating through the LK mechanism due to a stellar companion and friction due to tides. Although their initial conditions are different from ours, the fraction of migrating planets obtained in these studies and their orbital distribution are comparable to what is obtained in our study. For example, the fraction $(\sim 1 \%)$ and orbits of migrating planets displayed in Figure 10 of Petrovich (2015a) are clearly similar to those shown in our Figure 5. This suggests that while the perturber plays the fundamental role in inducing the planetary LK oscillations, the perturber properties are likely to not impact our general results. The main results of our paper should therefore apply also to the case in which the LK oscillations are induced by a stellar companion rather than an outer Jovian companion.

In conclusion, we have presented a numerical study of the dynamics of giant planets with close friends. We used both a secular code based on orbit-average equations of motion and direct three-body integrations to address whether the giant planets observed in the semimajor axis range 0.1-1 au could have been formed farther out and then migrated to these radii through secular migration processes such as LK cycles or secular chaos. The main results of our study are summarized below:

1. According to the high-e migration hypothesis, HJs and WJs formed originally at $\gtrsim 1$ au distance from their host star. In order to test this hypothesis, we addressed whether the observed Jupiter pairs hosting HJs and WJs would be dynamically stable if the inner planet was placed on an eccentric orbit (as required for efficient tidal dissipation) at $\gtrsim 1$ au. According to stability criteria that we have taken from the literature, only 4 of the 24 observed systems would be dynamically stable at these radii, with 14 of them being on intersecting orbits. As we also confirmed by direct integrations, if a planet pair is unstable, it does not lead to the formation of tidally migrating planets but rather to collisions with the host star or planet ejections. These findings argue against a high-e migration scenario from $\gtrsim 1$ au for the formation of most observed systems.

2. We showed that high-e migration models for WJs produce a period distribution that is not consistent with observations. By comparing the ratio of WJs to CJs we found in our simulations to that ratio as observed, we infer that $\lesssim 10 \%$ of all gas giants observed at $0.1-1$ au from their stellar host could have formed through high-e migration LK processes. Preliminary simulations of systems containing three to five planets suggest that the fraction of WJs produced in multiplanet systems is likely to be small as well.

3. Our analysis shows that high-e migration processes tend to produce more HJs than WJs. Accordingly, for any detected WJ with a close companion there should be at least an equal amount of detected HJs also with a close companion. In contrast with this prediction, and restricting ourself to Jupiters discovered through radial velocity surveys, we find that only two HJs have a detected outer giant companion within $\lesssim 5$ au, while 20 of the 74 period valley gas giants do. This points toward a different formation history for the two populations of planets.

4. Using both numerical and analytic techniques, we have shown that a tidal migration model produces an eccentricity distribution for the migrating planets that also appears to be inconsistent with observations. The oscillations required to produce inward migration tend to excite the eccentricities of migrating planets to values higher than those observed.

5. We showed that the observed eccentricity distribution of giant planets in the radial range $0.1-1$ au is consistent with the eccentricity distribution for planets at radii larger than $1 \mathrm{au}$. This might indicate a close relation between the two populations of planets and perhaps a common formation history.

Based on these results, we conclude that rather than starting on highly eccentric orbits with orbital periods above $1 \mathrm{yr}$, the Jupiters observed in the radial range 0.1-1 au from their stellar host are likely to have reached the region where they are observed today without tidal circularization. Tidal migration following planet-planet scattering is also disfavored given that we would not expect it to typically result in close and mildly eccentric companions to WJs (Nagasawa et al. 2008; Beaugé \& Nesvorný 2012).

Where do the WJs come from then? Our results may indicate that disk migration is the dominant channel for producing WJs. Alternatively, they might have formed in situ (Huang et al. 2016), i.e., they underwent runaway gas accretion, from originally low-mass closely packed planets (e.g., Lee et al. 2014). However, both disk migration and in situ formation are more likely to produce planets on nearly circular and low inclined orbits, so it remains to be explained how in these scenarios the planets' eccentricities could have been excited to the observed values. A possibility is that the eccentricities were excited through secular chaos (Wu \& Lithwick 2011), which could imply the presence of one or multiple still undetected planet companions to the numerous planet pairs observed in the period valley. ${ }^{3}$

We thank Bekki Dawson and Eugene Chiang for useful suggestions, and the referee for their detailed comments that helped to improve the paper. F.A. acknowledges support from a CIERA postdoctoral fellowship at Northwestern University.

\footnotetext{
3 As this paper was submitted, we became aware of a similar study by Petrovich \& Tremaine (2016). Based on a population synthesis study similar to ours, Petrovich \& Tremaine (2016) conclude that high-eccentricity migration excited by an outer planetary companion can account at most for $\sim 20 \%$ of the WJs, a fraction twice as large as the upper limit found here.
} 
A.S.H. was supported by the Netherlands Research Council NWO (grants 639.073.803 [VICI], 614.061.608 [AMUSE], and 612.071.305 [LGM]) and the Netherlands Research School for Astronomy (NOVA). Y.L. acknowledges grant AST1352369 from NSF and NNX14AD21G from NASA.

\section{REFERENCES}

Anderson, K. R., Storch, N. I., \& Lai, D. 2016, MNRAS, 456, 3671 Antonini, F., Murray, N., \& Mikkola, S. 2014, ApJ, 781, 45 Antonini, F., \& Perets, H. B. 2012, ApJ, 757, 27

Batygin, K., Bodenheimer, P. H., \& Laughlin, G. P. 2016, ApJ, 829, 114 Beaugé, C., \& Nesvorný, D. 2012, ApJ, 751, 119

Blaes, O., Lee, M. H., \& Socrates, A. 2002, ApJ, 578, 775

Boley, A. C., Granados Contreras, A. P., \& Gladman, B. 2016, ApJL, 817, L17

Correia, A. C. M., Udry, S., Mayor, M., et al. 2005, A\&A, 440, 751

Cumming, A. 2004, MNRAS, 354, 1165

Dawson, R. I., \& Chiang, E. 2014, Sci, 346, 212

Dawson, R. I., \& Murray-Clay, R. A. 2013, ApJL, 767, L24

Dawson, R. I., Murray-Clay, R. A., \& Johnson, J. A. 2015, ApJ, 798, 66

Desort, M., Lagrange, A.-M., Galland, F., et al. 2008, A\&A, 491, 883

Dong, S., Katz, B., \& Socrates, A. 2014, ApJL, 781, L5

Eggleton, P., \& Kiseleva, L. 1995, ApJ, 455, 640

Endl, M., Caldwell, D. A., Barclay, T., et al. 2014, ApJ, 795, 151

Frewen, S. F. N., \& Hansen, B. M. S. 2016, MNRAS, 455, 1538

Giguere, M. J., Fischer, D. A., Howard, A. W., et al. 2012, ApJ, 744, 4

Goldreich, P., \& Tremaine, S. 1980, ApJ, 241, 425

Haghighipour, N., Butler, R. P., Rivera, E. J., Henry, G. W., \& Vogt, S. S. 2012, ApJ, 756, 91

Hamers, A. S., Antonini, F., Lithwick, Y., Perets, H. B., \& Portegies Zwart, S. F. 2016, MNRAS, 464, 688

Hamers, A. S., Perets, H. B., Antonini, F., \& Portegies Zwart, S. F. 2015, MNRAS, 449, 4221

Hamers, A. S., \& Portegies Zwart, S. F. 2016, MNRAS, 459, 2827

Hébrard, G., Bonfils, X., Ségransan, D., et al. 2010, A\&A, 513, A69

Huang, C. X., Wu, Y., \& Triaud, A. H. M. J. 2016, ApJ, 825, 98

Hut, P. 1981, A\&A, 99, 126
Jones, H. R. A., Butler, R. P., Tinney, C. G., et al. 2010, MNRAS, 403, 1703

Lee, E. J., Chiang, E., \& Ormel, C. W. 2014, ApJ, 797, 95

Lin, D. N. C., Bodenheimer, P., \& Richardson, D. C. 1996, Natur, 380, 606

Mardling, R. A., \& Aarseth, S. J. 2001, MNRAS, 321, 398

Matsumura, S., Thommes, E. W., Chatterjee, S., \& Rasio, F. A. 2010, ApJ, 714, 194

Mayor, M., Udry, S., Naef, D., et al. 2004, A\&A, 415, 391

Merritt, D. 2013, Dynamics and Evolution of Galactic Nuclei (Princeton, NJ: Princeton Univ. Press)

Meschiari, S., Laughlin, G., Vogt, S. S., et al. 2011, ApJ, 727, 117

Mikkola, S., \& Merritt, D. 2006, MNRAS, 372, 219

Mikkola, S., \& Merritt, D. 2008, AJ, 135, 2398

Moorhead, A. V., Ford, E. B., Morehead, R. C., et al. 2011, ApJS, 197, 1

Moutou, C., Hébrard, G., Bouchy, F., et al. 2014, A\&A, 563, A22

Nagasawa, M., Ida, S., \& Bessho, T. 2008, ApJ, 678, 498

Naoz, S., Farr, W. M., Lithwick, Y., Rasio, F. A., \& Teyssandier, J. 2011, Natur, 473, 187

Niedzielski, A., Villaver, E., Wolszczan, A., et al. 2015, A\&A, 573, A36

Petrovich, C. 2015a, ApJ, 799, 27

Petrovich, C. 2015b, ApJ, 808, 120

Petrovich, C., \& Tremaine, S. 2016, ApJ, 829, 132

Pilyavsky, G., Mahadevan, S., Kane, S. R., et al. 2011, ApJ, 743, 162

Quinn, S. N., White, T. R., Latham, D. W., et al. 2015, ApJ, 803, 49

Rasio, F. A., \& Ford, E. B. 1996, Sci, 274, 954

Robertson, P., Endl, M., Cochran, W. D., et al. 2012, ApJ, 749, 39

Santerne, A., Moutou, C., Tsantaki, M., et al. 2016, A\&A, 587, A64

Ségransan, D., Udry, S., Mayor, M., et al. 2010, A\&A, 511, A45

Socrates, A., Katz, B., \& Dong, S. 2012a, arXiv:1209.5724

Socrates, A., Katz, B., Dong, S., \& Tremaine, S. 2012b, ApJ, 750, 106

Tan, X., Payne, M. J., Lee, M. H., et al. 2013, ApJ, 777, 101

Tinney, C. G., Butler, R. P., Marcy, G. W., et al. 2006, ApJ, 647, 594

Wang, X., Sharon, Wright, J. T., et al. 2012, ApJ, 761, 46

Winn, J. N., Johnson, J. A., Howard, A. W., et al. 2010, ApJ, 718, 575

Wright, J. T., Fakhouri, O., Marcy, G. W., et al. 2011, PASP, 123, 412

Wright, J. T., Marcy, G. W., Howard, A. W., et al. 2012, ApJ, 753, 160

Wright, J. T., Upadhyay, S., Marcy, G. W., et al. 2009, ApJ, 693, 1084

Wu, Y., \& Lithwick, Y. 2011, ApJ, 735, 109

Wu, Y., \& Murray, N. 2003, ApJ, 589, 605 